\title{
An Analysis of Topological Ring-Currents and their Use in Assessing the Annulene-Within-an-Annulene Model for Super-Ring Conjugated Systems ${ }^{\dagger}$
}

\author{
T. K. Dickens ${ }^{\mathrm{a}}$ and R. B. Mallion ${ }^{\mathrm{b}, *}$ \\ ${ }^{\mathrm{a}}$ University Chemical Laboratory, University of Cambridge, Lensfield Road, Cambridge, \\ CB2 1EW, England, United Kingdom \\ ${ }^{\mathrm{b}}$ School of Physical Sciences, University of Kent, Canterbury CT2 7NH, England, United Kingdom
}

RECEIVED MAY 14, 2013; REVISED AUGUST 10, 2013; ACCEPTED AUGUST 12, 2013

\begin{abstract}
The Hückel-London-Pople-McWeeny approach to calculating ring currents in conjugated systems is considered in detail, especially the 'topological' variant of this formalism, particularly emphasised during the last five years. A step-by-step account is given of how these calculations are effected in practice, in such a way as to enable any interested reader to carry out these computations himself. The method is illustrated by using it to assess the applicability of the so-called 'Annulene-Within-an-Annulene (AWA) Rule' to 'super-ring' conjugated hydrocarbons - some in which the inner ring and outer perimeter are 'coupled', and others in which they are 'decoupled'. Compliance with the AWA model appears more probable in decoupled super-ring structures than in coupled ones. The Appendix touches on previous work involving Professor D. J. Klein, the honorand of this issue. (doi: 10.5562/cca2291)
\end{abstract}

Keywords: topological ring-currents and bond-currents, graph theory , conjugated hydrocarbons, coupled and decoupled super-ring systems, annulene-within-an-annulene rule

\section{INTRODUCTION}

In this paper, a detailed re-consideration is presented of the Hückel ${ }^{1}$-London ${ }^{2}$-Pople ${ }^{3}$-McWeeny ${ }^{4}$ (HLPM) approach to calculating ring-currents and bond currents in conjugated systems (see Refs. 5-8 for reviews), in which a step-by-step account is given of how these calculations are in practice carried out, with special reference to the 'topological' variant of this formalism, recently emphasised. ${ }^{9-11}$ Application of this approach will be illustrated by using it to compute numerical values of ring currents and bond currents in order to re-enforce a recent re-evaluation ${ }^{12,13}$ of the 'AnnuleneWithin-an-Annulene' (AWA) model, ${ }^{14-24}$ a model which has been of interest to several groups of workers, ${ }^{14-24}$ especially over the last decade or so. ${ }^{16-24}$

The simplicity of this HLPM topological formalism is that, unlike with most other methods for calculating ring currents, there is no requirement to determine atomic coordinates in order to perform these calculations. All that is required is the connectivity matrix and the geometric areas of the constituent rings, and we choose to perform the calculation in two stages - a purely topological stage, followed by one that involves the actual geometry of the conjugated system. Furthermore, this approach requires no empirical parameters. The ring currents themselves are expressed as a ratio to the ring-current intensity calculated, by the same method, for benzene: this practice has the dual benefits of ensuring that the resulting calculated ring-currents are dimensionless, and of being an aid to simple comparison between different ring-systems as well as with the predictions from other methods of calculation (e.g. Ref. 24).

The AWA model arises in the context of conjugated systems that consist of a central ring of carbon atoms joined by 'transverse, 25 ('spokes') ${ }^{21}$ bonds to a larger, outer ring, as in Figure 1. Depending on the sizes of these two rings, four distinct cases can arise: a [4n]annulene-within-a-[4m]-annulene, a [4n]-annulenewithin-a-[4m+2]-annulene, a [4n+2]-annulene-withina-[4m]-annulene, and a $[4 n+2]$-annulene-within-a[ $4 m+2]$-annulene (where $n$ and $m$ are positive integers, in every case). Such entities have been called 'superring' structures. ${ }^{26,27}$

It has long been known ${ }^{28-31}$ that annulenes - and we refer here strictly to the classic, monocyclic annulenes - are associated with paramagnetic $\pi$-electron

\footnotetext{
$\dagger$ Dedicated to Professor Douglas Jay Klein on the occasion of his $70^{\text {th }}$ birthday.

* Author to whom correspondence should be addressed. (E-mail: R.B.Mallion@kent.ac.uk)
} 


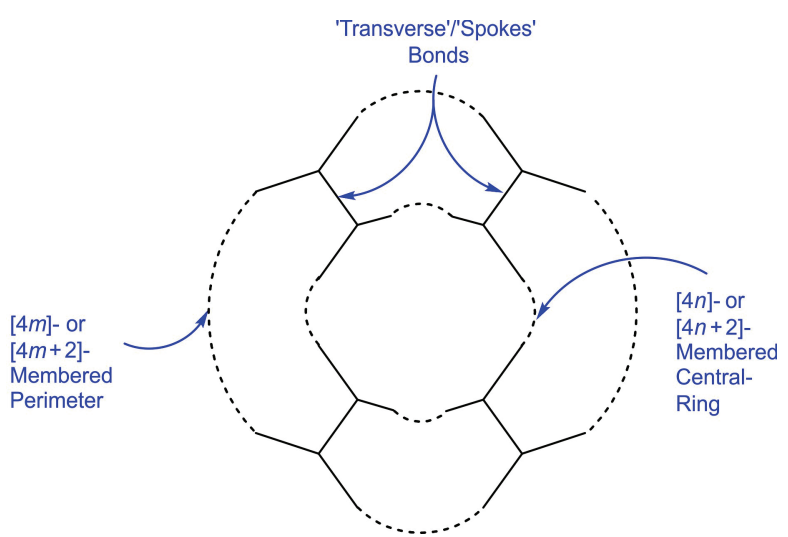

Figure 1. Schematic depiction of a general 'super-ring' structure, ${ }^{26,27}$ showing the central ring ([4n]- or $[4 n+2]$ membered), the outer perimeter ([4m]- or [ $4 m+2]$-membered), and the "transverse ${ }^{25 /}$ 'spokes ${ }^{21}$ bonds that connect the inner ring and the outer perimeter.

currents if they consist of [4n] carbon-atoms and with diamagnetic currents if the size of their (unique) ring may be expressed in the form [ $4 n+2]$; (see reference 44 of Ref. 23 for a detailed bibliography supporting this assertion). A given super-ring structure ${ }^{26,27}$ is said to obey the AWA model ${ }^{14-24}$ if both its central ring and its outer perimeter bear calculated $\pi$-electron currents according to the pattern described above - implying that the inner and outer rings effectively behave as if they were independent annulenes. Most super-ring ${ }^{26,27}$ structures seem, however, to violate the AWA model when tested against the results of ab-initio ipso-centric calculations $^{17-21,32,33}$ and of 'pseudo- $\pi$ ' calculations ${ }^{34}$ of the type frequently presented by Fowler and co-workers, and also when confronted with topological ${ }^{9-11}$ $\pi$-electron ring-currents and bond-currents that we ourselves have calculated ${ }^{22,23}$ by means of the HLPM approach. $^{1-4}$

By contrast, the present authors have recently shown $^{12,13}$ that, in a homologous series called the $p$-coronenes, ${ }^{12}$ in which the inner ring and outer perimeter are 'decoupled' in the sense defined by Fowler et al., ${ }^{17-21}$ the AWA rule is respected - at least, when HLPM 'topological' bond-currents are used as the criterion. $^{12}$ (It should however be noted that this conclusion is not supported by some very recent calculations ${ }^{24}$ based on the 'ipso-centric' ab-initio approach). ${ }^{17-21,32,33}$ The AWA model is likewise complied with ${ }^{13}$ by the one member of the regular $[r, s]$-coronene series ${ }^{25}$ in which the outer and inner rings are 'decoupled', but not by other regular $[r, s]$-coronenes, ${ }^{25}$ in which these two sets of rings are 'coupled'. ${ }^{13}$

The present account further evaluates the possible connection between the decoupled or coupled nature ${ }^{17-21}$ of the outer perimeter and inner ring and compliance with, or violation of, the AWA rule. We do this in the first instance by considering, as exemplars, the seven (coupled) super-ring structures that were studied by

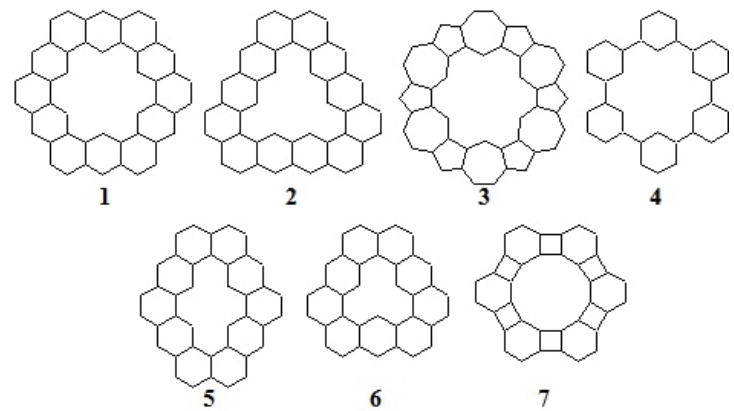

Figure 2. The carbon-atom skeletons of the seven 'super-ring' structures considered by Aihara in Ref. 27.

Aihara in Ref. 27 (all of which are illustrated in Figure 2). In the course of this, because the process has so far been only partially documented elsewhere, $, 10,35,36$ we take the opportunity here to describe explicitly how a topological $^{9-11}$ calculation of $\pi$-electron ring-currents and bond-currents is actually carried out in practice, when the computations are effected by means of the HLPM method. ${ }^{1-4}$ The full details of a calculation of this type are presented in such a way as to enable any interested reader actually to carry out a ring-current computation by this method, if desired.

\section{CALCULATIONS}

It is well-known that energy levels calculated via Hückel theory, ${ }^{1}$ and the LCAO molecular-orbitals that are associated with them, are purely topological in nature - that is to say, they depend solely on how the carbon-atoms of the network under study are connected to each other in the $\sigma$-bond framework of that conjugated structure; (see Refs. 37-41 for reviews). Calculating a $\pi$-electron ring-current in such a system by means of the HLPM ${ }^{1-4}$ method does, however, involve knowledge of molecular geometry, ${ }^{36}$ as well as of the carboncarbon connectivity extant in the system's molecular graph $^{37-41}$ (such as is displayed for structures 1-7 in Figure 2). Consequently, a $\pi$-electron ring-current may be calculated directly from a single equation by means of modifications to the original London method ${ }^{2}$ that simultaneously incorporate both these geometrical and topological aspects: for example, one due to $\mathrm{Salem}^{42}$ (following Berthier) ${ }^{43}$ states that (for a closed-shell system):

$$
J_{i}=-\frac{2 e}{h} \sum_{K=1}^{\mathrm{occ}} \frac{\partial E_{K}}{\partial f_{i}}
$$

in which the summation runs over all occupied orbitals (which are assumed to be doubly occupied) and where $J_{i}$ is the ring-current intensity in the $i^{\text {th }}$ ring of the conjugated system, $E_{K}$ is the ordinary $K^{\text {th }}$ Hückel energy-level in the absence of a magnetic field, $e$ is the 
charge on the electron, $h$ is Planck's constant, and $f_{i}$ is given by

$$
f_{i}=\frac{e}{h} B_{0} s_{i}
$$

in which $B_{0}$ is the size of the magnetic field (considered to be applied at right angles to the molecular plane), and $s_{i}$ is the (signed) area of the $i^{\text {th }}$ ring (counted positive if the ring's perimeter is traversed in the anti-clockwise sense about the upward normal through that ring's centre, and negative if the traversal is effected in the clockwise direction). The quantity $f_{i}$ is thus proportional to the magnetic flux, due to the external magnetic-field, through the area of the $i^{\text {th }}$ ring of the conjugated system. An equivalent expression was given by Pople, in his seminal paper ${ }^{3}$ of 1958 . More recently, Fowler and Myrvold $^{44}$ have cast the above expression for the ring current for closed-shell systems in terms of bond currents, $J_{k l}$, in the individual bonds, $k l$, as follows

$$
J_{k l}=-\frac{2 e}{h} \sum_{K=1}^{\mathrm{occ}} \frac{\partial E_{K}}{\partial f_{k l}}
$$

where

$$
f_{k l}=\frac{e}{h} B_{0} s_{k l}
$$

in which $s_{k l}$ is the signed area ${ }^{45}$ of the triangle formed by atoms $k$ and $l$ and the origin, $\mathrm{O}$ (in the molecular plane; if the origin is not in the molecular plane, then the projection, $s_{k l}^{\prime}$, of the area of the triangle $\mathrm{O} k l$, into the molecular plane, must replace $\left.s_{k l}\right) .{ }^{46,47}$ The sets of ring currents, $\left\{J_{i}\right\}$, and bond currents, $\left\{J_{k l}\right\}$, are connected to each other by their mutual compliance with the microscopic analogy of Kirchhoff's Law of Current Conservation at a Junction in macroscopic electrical circuits. ${ }^{6,35,36,48-50}$

Overtly graph-theoretical ${ }^{37-41}$ versions of the Hückel-London ${ }^{1,2}$ approach have since been provided. A much-neglected one, by Mitzoguchi, ${ }^{51}$ elegantly exploited the Coulson contour-integral ${ }^{52}$ version of Hückel theory and applied it to diamagnetic susceptibilities - quite independently of Coulson himself, who had privately ${ }^{53}$ investigated these matters (using his own contour-integral method ${ }^{52}$ ) as early as 1953; Coulson did this by devising an approach that also involved polarisabilities (which thus has interesting parallels to the McWeeny formalism ${ }^{4}$ of five years later, which is the approach that the present authors will be concentrating on in this study). The intriguing story of Coulson's 'private' contributions to this field is told in Ref. 45. Another graph-theoretical formalism was proposed by
Aihara and co-workers, ${ }^{54-57}$ who presented a form of the above equations couched in terms of graph-theoretical characteristic-polynomials, ${ }^{38,39} P_{G}(E)$, of the molecular graph, $G$, representing the conjugated network under study, and of graphs obtained from it by appropriately deleting certain circuits, $r_{k}$. Thus: ${ }^{55-57}$

$$
\frac{I_{k}}{I_{\text {benzene }}}=18\left(\frac{c_{k}}{c_{\text {benzene }}}\right) \sum_{K}^{\text {occ }} \frac{P_{G-r_{k}}\left(E_{K}\right)}{P_{G}^{\prime}\left(E_{K}\right)}
$$

In equation (5), $I_{k}$ is the current associated with the $k^{\text {th }}$ circuit, $r_{k}$, within $G$ and $I_{\text {benzene }}$ is the corresponding current around the single circuit in benzene. Furthermore, $c_{k}$ and $c_{\text {benzene }}$ are the areas of the circuit $r_{k}$ and of a standard benzene-ring, respectively, $G-r_{k}$ is the graph remaining when the circuit $r_{k}$ is deleted from $G$, and and $P_{G}$ and $P_{G-r_{t}}(E)$ are the characteristic polynomials $^{38,39}$ of the graphs $G$ and $G-r_{k}$, respectively; $P_{G}^{\prime}(E)$ is the derivative, with respect to $E$, of $P_{G}(E) . E_{K}$ is again the $K^{\text {th }}$ largest zero of $P_{G}(E)$ and the summation runs over all occupied molecular-orbitals, $K$, which are assumed to be doubly occupied. (If $P_{G}(E)$ has repeated roots, this formula must be replaced by others. $)^{53,54,56}$ The ring-current intensity in the $i^{\text {th }}$ ring, relative to benzene $\left(J_{i} / J_{\text {benzene }}-c f\right.$. equation (1)), is then simply obtained by (algebraically) adding the quantities $I_{k} / I_{\text {benzene, }}$ computed from equation (5), over all circuits, $r_{k}$, that contain a specified ring, $i$, in which the ring current is required. ${ }^{54-57}$

McWeeny's work ${ }^{4}$ was simultaneous with, and independent of, that of Pople. ${ }^{3}$ However, McWeeny ${ }^{4}$ chose not to give a general explicit expression for the ring-current intensity in individual rings of a general polycyclic system when describing his (equivalent) representation of the London method ${ }^{2}$ in his own (likewise seminal) paper, also published in the $1958 \mathrm{Molecu}$ lar Physics. ${ }^{4}$ One of us has, however, shown that this is easily done, in the context of the McWeeny method, ${ }^{4}$ if required. ${ }^{35,36}$ This expression will eventually be presented as equation (14) of the present paper.

Ring-current and bond-current intensities may thus be computed from a single equation, (such as (1) and (3), above, and from equation (5) when the appropriate summations over circuits have been performed). These involve both geometrical and topological aspects. The present authors, however, choose to do the calculation in two distinct and separate stages:

(a) one (the Hückel calculation) that computes just the topological quantities required; and

(b) a second stage, which involves combining the output from (a) with geometrical data about the structure, which depends explicitly on the areas of its constituent rings.

We now consider these two stages separately. 
(a) (i) The Hückel Calculation

The vertices of the molecular graph gr-41 $^{37}$ are arbitrarily labelled and a standard Hückel calculation ${ }^{1}$ is carried out, with all Coulomb integrals, $\alpha$, set equal to the standard value in benzene; furthermore, when a 'topological' calculation is being performed, all resonance integrals between pairs of bonded carbon-atoms are likewise assigned the standard benzene value, $\beta$. In graph-theoretical terminology, ${ }^{37-41}$ we are effectively finding the set of eigenvectors, $\left\{\mathbf{c}_{J}\right\}_{J=1,2, \ldots, N}\left(=\left\{\left(c_{J 1}\right.\right.\right.$ $\left.\left.\left.c_{J 2}, \ldots, c_{J N}\right)^{T}\right\}_{J=1,2, \ldots, N}\right)$, and the family of eigenvalues, $\left\{E_{J}\right\}_{J}=1,2, \ldots, N$, of the vertex-adjacency matrix ${ }^{40}$ of the associated molecular-graph ${ }^{39}$ which, in general, is taken to have $N$ vertices. Because the energy of the $J^{\text {th }}$ molecular-orbital is $\left(\alpha+\beta E_{J}\right)$ and $\beta$ is negative, the eigenvalues are conventionally labelled so that $E_{1} \geq E_{2} \geq \ldots \geq E_{N}$ The corresponding molecular-orbital energy-levels are thereby labelled in increasing order as is required for an application of the Aufbau process (as next described).

(ii) A $\pi$-Electron Ground-State Configuration Obtained via the Aufbau Process

Application of the Aufbau Principal determines a set of values for the eigenvalue/energy-level occupationnumbers $\left\{v_{J}\right\}_{J=1,2, \ldots, N}$. The occupation number, $v_{J}$, is 2 if the $J^{\text {th }}$ molecular-orbital (i.e., the $J^{\text {th }}$-largest eigenvalue of the molecular graph) is doubly occupied when the Aufbau process ${ }^{41,58-60}$ has been applied, $v_{J}=1$ if the $J^{\text {th }}$ molecular-orbital is singly occupied, and $v_{J}=0$ if the $J^{\text {th }}$ molecular-orbital is entirely unoccupied by electrons. It has been shown ${ }^{41,58-60}$ that, once the family of eigenvalues, $\left\{E_{J}\right\}_{J=1,2, \ldots, N}$, of the vertex-adjacency matrix ${ }^{40}$ of the associated molecular-graph ${ }^{39}$ has been established, application of the Aufbau procedure ${ }^{41,58-60}$ to determine $\left\{v_{J}\right\}_{J=1,2, \ldots, N}$, - that is, a ground-state electronic configuration for the conjugated system in question is likewise an entirely topological process that depends solely on the relative order of the eigenvalues, $\left\{E_{J}\right\}_{J=1,2, \ldots, N .^{41,58-60}}$

(iii) Coulson Bond-Orders and Imaginary Self- and Mutual Bond-Bond Polarisabilities Calculated from (i) the Hückel Quantities and (ii) the $\pi$-Electron GroundState Configuration

Starting from knowledge of (a) the set of eigenvectors, $\left\{\mathbf{c}_{J}\right\}_{J}=1,2, \ldots, N$, and the family of eigenvalues, $\left\{E_{J}\right\}_{J}=1,2, \ldots, N$, of the vertex-adjacency matrix ${ }^{40}$ of the associated molecular-graph, ${ }^{39}$ and (b) the occupation numbers, $\left\{v_{J}\right\}_{J}=1,2, \ldots, N$, devised by an application $^{41,58-60}$ of the Aufbau Principle, it is straightforward to define

$(\alpha)$ The Coulson Bond-Order, ${ }^{1,61} P_{(r s)}$, of the bond joining the carbon-atoms labelled ' $r$ ' and ' $s$ ' in the associated molecular-graph

$$
P_{(r s)}=\sum_{J=1}^{N} v_{J} c_{J r} c_{J s}
$$

( $\beta$ ) The (real) mutual bond-bond polarisability, $\pi_{(r s)(t u)}$, between bonds $(r s)$ and $(t u)$, which is ${ }^{62}$ the rate at which the real part of the bond-order, $P_{(r s)}$, of the bond (rs) changes with respect to a variation in the real part of the resonance integral, $\beta_{(t u)}$, of the bond (tu).

$$
\pi_{(r s)(t u)}=\frac{\partial P_{(r s)}}{\partial \beta_{(t u)}}
$$

This, in its turn, can be defined ${ }^{4}$

$$
\pi_{(r s)(t u)}=\pi_{r s, t u}+\pi_{r s, u t}+\pi_{s r, u t}+\pi_{s r, t u}
$$

The individual entities on the right-hand side of equation (8) are defined as follows ${ }^{4,36}$

$$
\pi_{r s, t u}=\frac{2}{\beta} \sum_{I=1}^{M} \sum_{J=M+1}^{N} \frac{c_{I r} c_{J S} c_{J t} c_{I u}}{E_{I}-E_{J}}
$$

where the orbitals 1 to $M$ are (doubly) occupied.

$(\gamma)$ The imaginary mutual bond-bond polarisability, $\bar{\pi}_{(r s)(t u)}$, between bonds $(r s)$ and $(t u)$ - the imaginary aspect arising as a result of the influence of the external magnetic-field ${ }^{4}-$ is $^{4}$ the rate at which the imaginary part of the bond-order, $P_{(r s)}$, of the bond $(r s)$ changes with respect to a variation in the imaginary part of the resonance integral, $\beta_{(t u)}$, of the bond $(t u)$. In terms of the quantities defined in equation (9), this is ${ }^{4}$

$\bar{\pi}_{(r s)(t u)}=\pi_{r s, t u}-\pi_{r s, u t}+\pi_{s r, u t}-\pi_{s r, t u}$

The following relations hold: ${ }^{4}$

$$
\bar{\pi}_{(r s)(t u)}=-\bar{\pi}_{(r s)(u t)}=-\bar{\pi}_{(s r)(t u)}=\bar{\pi}_{(s r)(u t)}
$$

The above determines that, because this really is a mutual imaginary bond-bond polarisability, the order in which the two bonds are mentioned is immaterial, and the following statement is true:

$$
\bar{\pi}_{(r s)(t u)}=\bar{\pi}_{(t u)(r s)}
$$

Finally, if bonds $(r s)$ and $(t u)$ are the same bond, we have the imaginary self bond-bond polarisability, $\bar{\pi}_{(r s)(r s)}$ :

$$
\bar{\pi}_{(r s)(r s)}=\pi_{r s, r s}-\pi_{r s, s r}+\pi_{s r, s r}-\pi_{s r, r s}
$$


<smiles></smiles><smiles>CCCCCCCCCCCCCCCCCCCCCCCCCCC</smiles>

Figure 3. The molecular graph of coronene (left), and (right) a continuous (un-branched) spanning-tree of that molecular graph.

(b) (i) Selection of an Un-branched Spanning-Tree, Establishment of a Fundamental System of Circuits for that Spanning Tree, and Use of the McWeeny UnitaryTransformation on the Basis Orbitals. Calculation of Ring Currents

The process of calculating a ring-current by the HLPM approach ${ }^{1-4,9-11,35,36}$ is here illustrated by using coronene as an example. The arbitrarily labelled molecular-graph of coronene is shown on the left-hand side of Figure 3.

A chemist would say that coronene is a structure which has seven rings; a mathematician would say that its carbon-carbon connectivity is a graph with circuitrank 7. If an edge (bond) is judiciously removed from each ring in such a way as to destroy all what might intuitively be called 'closed loops' of carbon atoms, the graph that ultimately remains would still be connected but would no longer contain any closed circuits of vertices (carbon-atoms). Such a graph is called a spanning tree of the original graph, ${ }^{63}$ and an example is depicted on the right-hand side of Figure 3 . There are a large number of ways in which this can be done. In the Appendix it is shown that there are in fact precisely 176400 such spanning-trees for the coronene moleculargraph (the example being considered here). Because the coronene molecular-graph is planar (that is, it can be drawn in a plane - for example, as in Figure 3 without requiring any edges to cross over each other) this result is obtained in the Appendix by an application of the theorem of Gutman et al. ${ }^{64}$ (1983), which is a special case (applicable only to such planar graphs) of the more-general 'Cycle Theorem' (2004) due to D. J. Klein, the honorand of this issue of Croatica Chemica Acta, and others (including one of us [RBM]). ${ }^{65}$ In the Appendix this value of 176400 for the number of spanning trees in the coronene molecular-graph is also confirmed by an application of the traditional Matrix Tree Theorem. ${ }^{63}$ However, the motto here is: 'one is enough' - only a single one of these 176400 spanningtrees is actually required in practice in order to able to effect the ring-current calculation that is being carried out here.
In his original treatment, McWeeny ${ }^{4}$ prescribed that the chosen spanning-tree on which the ring-current calculation is to be based must be what he described as 'any chain which is continuous and open (i.e. without loops)' - that is to say, it must be a tree that represents what a graph theorist would call a semi-Hamiltonian path $^{66,36}$ through the network represented by the molecular graph. Such spanning trees will be referred to as 'un-branched' (or as 'continuous') spanning-trees, and an example is depicted on the right-hand side of Figure 3. McWeeny also (arbitrarily) prescribed ${ }^{4}$ that the vertices of the 'continuous and open' chain shall be numbered consecutively — as indeed is done in Figure 3.

Having obtained a spanning tree such as that on the right-hand side of Figure 3, it would then be possible to insert back into it one of the removed edges - let us call it edge No (1) — as in the upper-left part of Figure 4. This would create a unique circuit, as shown there. If edge (1) were again removed and then edge (2) (only) were re-inserted instead, another unique circuit would be created (as is illustrated in the second drawing in the first row of Figure 4). If this process were continued by subsequently inserting (separately, and one-at-atime) the edges (3), (4), (5), (6) and (7), the unique circuits shown in the last five parts of Figure 4 would be created. The edges that were removed to form the spanning tree were called by McWeeny 'the bonds which complete circuits in the molecule'; graph-theoretically they are referred to as 'chords'. ${ }^{63}$ The set of (in this case) seven circuits so-formed when the seven chords are re-inserted back into the spanning tree in the process just described is called a Fundamental System of Circuits $^{63}$ (of, in this case, the coronene molecular-graph, depicted on the left-hand side of Figure 3).

It may be noted in passing that the above process (imagined as having been effected at the microscopic, molecular level) was exactly the one that Kirchhoff invoked when enunciating his Laws of Electrical Circuits in a macroscopic network, ${ }^{67,68}$ as long ago as 1845-1847.

The 'circuit-completing bonds'/'chords' are defined with an (arbitrary) direction assigned to them. Following McWeeny, ${ }^{4}$ the present authors routinely adopt the convention that such bonds/chords are defined from the carbon atom (vertex) of the numerically higher label to that of the numerically lower label. (For example, chord (1) in Figure 4 is defined in the direction ' $6 \rightarrow 1$ '.) However, the opposite (or no) convention would have been equally suitable for our purposes.

Having chosen an un-branched ('continuous') spanning-tree as described, and established the Fundamental System of Circuits associated with it, McWeeny then proposed what the present authors consider to be one of the most elegant applications of Hückel Theory: 

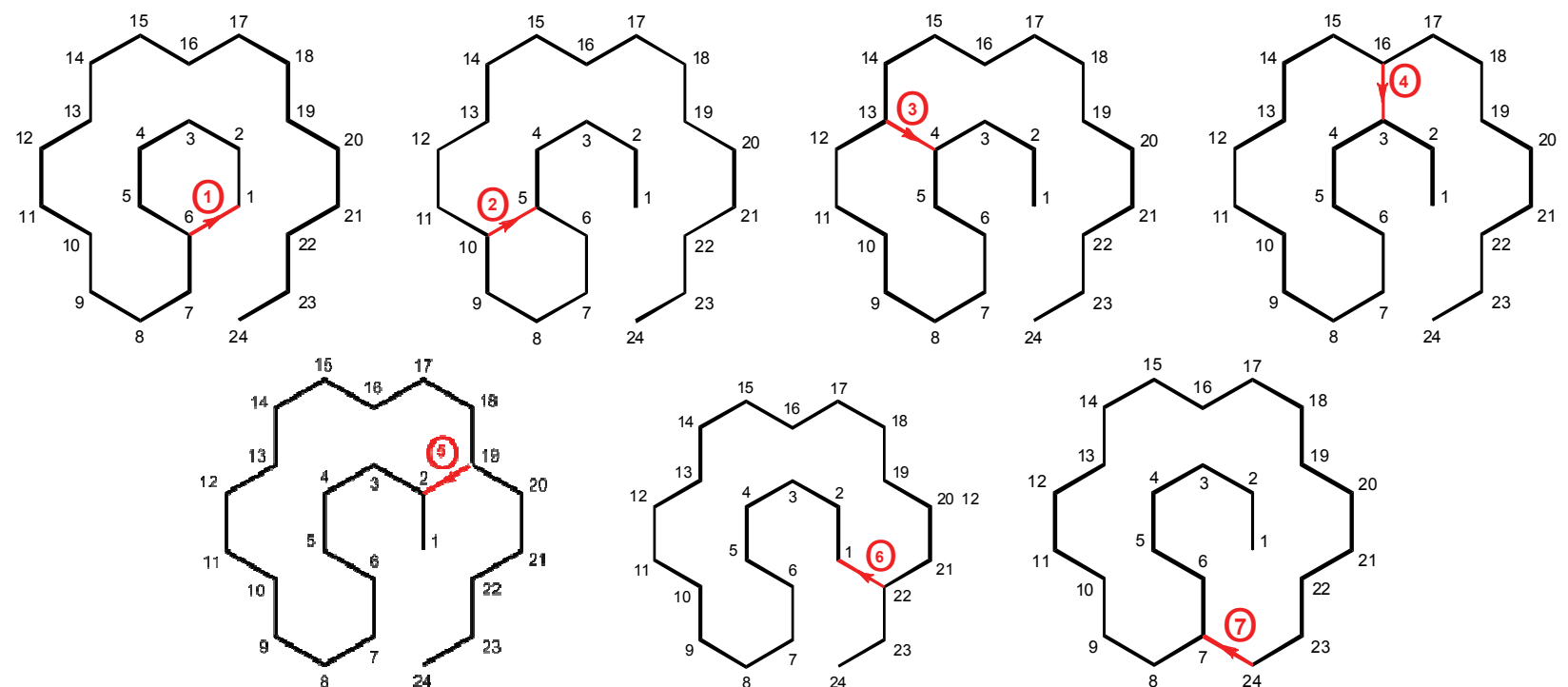

Figure 4. The Fundamental System of Circuits ${ }^{63}$ associated with the continuous (un-branched) spanning-tree (shown on the righthand side of Figure 3 ) of the coronene molecular-graph (depicted on the left-hand side of Figure 3 ).

McWeeny devised a clever unitary transformation ${ }^{4}$ on the basis orbitals in such a way that all the perturbation brought about by the presence of the external magneticfield is concentrated into just the circuit-completing bonds. As a result of this transformation, all the other off-diagonal elements of the Hückel Hamiltonian revert to their 'field-free' form - that is, they are precisely what they would be in the absence of any external magnetic-field. As McWeeny ${ }^{4}$ stated, this result '...shows at once that the perturbations to be applied depend on the presence of closed circuits and that without such circuits the field has no effect'.

With that preparation, McWeeny was able to apply what will henceforth be referred to as McWeeny's 'Master Equation', as follows

$$
\left(\frac{J_{i}}{J_{\text {benzene }}}\right)=9\left\{\begin{array}{l}
\sum_{(\mu)}\left[P_{(\mu)}+\beta \bar{\pi}_{(\mu)(\mu)}\right] S_{(\mu)} C_{(\mu)}^{i}+ \\
\sum_{(\mu<v)(v)} \sum_{(\nu)} \beta \bar{\pi}_{(\mu)(v)}\left[S_{(\mu)} C_{(v)}^{i}+C_{(\mu)}^{i} S_{(v)}\right.
\end{array}\right\}
$$

In fact, McWeeny did not explicitly write this equation in this way; casting the ring-current intensity in the $i^{\text {th }}$ ring in this form is due to one of the present authors (RBM), some years later. ${ }^{35,36}$ In the above simplified form (suitable for calculating what the present authors refer to as 'topological' ring-current intensities ${ }^{9-11}$ ) it is as equation (15) of Ref. 36, and, in a more-general form - based on Veillard's very aesthetic but seldomcited extension ${ }^{69}$ of McWeeny's formalism ${ }^{4}$ to enable its application to hetero-cyclic structures (see, for example, Refs. 70 and 71) - it is as presented as equation (25) in Ref. 35.
In this 'Master Equation' (14), $J_{i}$ is the ringcurrent intensity in the $i^{\text {th }}$ ring of the conjugated system and $J_{\text {benzene }}$ is the corresponding ring-current intensity calculated, by the same method, for benzene. It is immediately seen, therefore, that the quantities $\left(J_{i} / J_{\text {benzene }}\right)$ are dimensionless, without units. The double summations in the above equation are over all combinations of pairs of 'circuit-completing bonds', $\mu$ and $v$ (with $\mu<v$ ). $\beta$ is the standard Hückel resonance-integral, ${ }^{1} P_{(\mu)}$ is the Coulson bond-order, ${ }^{1,61}$ previously defined in equation (6), and the quantities $\beta \bar{\pi}_{(\mu)(\mu)}$ and $\beta \bar{\pi}_{(\mu)(v)}$ are (respectively) ( $\beta$ multiplied by) the self- and mutual imaginary bond-bond polarisabilities of circuit-completing bonds $\mu$ and $v$ (also previously defined, in equations (9), (10) and (13)). All of these quantities may be calculated (from the equations just stated) solely from a knowledge of the molecular graph ${ }^{37-41}$ of the conjugated system in question, via the eigenvalues and eigenvectors of its adjacency matrix, which are latent in the structure and which are, accordingly, pre-determined as soon as the carbon-atom adjacencies are specified. ${ }^{9,36}$ It should also be noted that $P_{(\mu)}, \beta \bar{\pi}_{(\mu)(\mu)}$, and $\beta \bar{\pi}_{(\mu)(v)}$ are calculable solely from knowledge of that molecular graph, without recourse to any parameters. $S_{(\mu)}$, is the signed (algebraical) area of the $\mu^{\text {th }}$ circuit - i.e., the enclosed area formed when the $\mu^{\text {th }}$ circuit-completing bond, only, is inserted into the spanning tree on which the calculation is being based..$^{4-6,9,11,35,36}$ These areas are to be counted positive if the arrow on the $\mu^{\text {th }}$ circuitcompleting bond (which, as already observed, bears an arbitrarily assigned direction) points in the anticlockwise sense around the circuit that it completes (the $\mu^{\text {th }}$ circuit), and are to be counted negative if that arrow points in the clockwise sense around the circuit that the 
Table 1. Coulson bond-orders for the $\mu^{\text {th }}$ circuit-completing bond (middle column) and algebraical areas for the $\mu^{\text {th }}$ fundamental circuit (right-hand column) $(\mu=1,2, \ldots, 7)$ for the 'continuous' ('un-branched') spanning-tree of the coronene molecular-graph depicted on the right-hand side of Figure 3

\begin{tabular}{ccc}
\hline $\begin{array}{c}\text { Labelling }(\mu) \text { of } \\
\text { fundamental } \\
\text { circuit (see } \\
\text { Figure 4) }\end{array}$ & $\begin{array}{c}\text { Coulson bond- } \\
\text { order of } \\
\mu^{\text {th }} \text { circuit-closing } \\
\text { bond }\end{array}$ & $\begin{array}{c}\text { Signed area of } \\
\mu^{\text {th }} \text { fundamental } \\
\text { circuit (see } \\
\text { Figure 4) }\end{array}$ \\
\hline (1) & 0.52189 & 1.00000 \\
(2) & 0.53802 & -1.00000 \\
(3) & 0.53802 & -2.00000 \\
(4) & 0.53802 & -3.00000 \\
(5) & 0.53802 & -4.00000 \\
(6) & 0.53802 & -5.00000 \\
(7) & 0.53802 & -7.00000 \\
\hline
\end{tabular}

$\mu^{\text {th }}$ circuit-closing bond completes. The quantities $C_{(\mu)}{ }^{i}$ (effectively the elements of a $(\mu \times \mu)$ matrix comprising only the entries $0,+1$ and -1 ) are likewise purely topological in nature and take on the values $0,+1$ or -1 according to whether (respectively) (a) the $i^{\text {th }}$ ring does not lie within the $\mu^{\text {th }}$ circuit, (b) the $i^{\text {th }}$ ring lies within the circuit completed by the $\mu^{\text {th }}$ circuit-completing bond and that circuit-completing bond is directed in the anticlockwise sense around the circuit (the $\mu^{\text {th }}$ one) that it completes, (c) the $i^{\text {th }}$ ring lies within the $\mu^{\text {th }}$ circuit but the $\mu^{\text {th }}$ circuit-completing bond points in the clockwise direction around the $\mu^{\text {th }}$ circuit. (For example, from Figure 4 , it can easily be verified by inspection that $C_{(1)}$ Central Ring $=+1, C_{(2)}$ Central Ring $=0$, and $C_{(7)}$ Central Ring $=$ -1 .)

It may be noted in passing that, in the special case of the mono-cyclic reference-structure, benzene, all the summations over pairs of circuit-completing bonds on the right-hand side of equation (14) collapse simply to

$$
\left[P_{\text {(benzene) }}+\beta \bar{\pi}_{\text {(benzene)(benzene) }}\right] S_{\text {(benzene) }} C_{\text {(benzene) }}^{1}
$$

In expression (15), when areas are measured in benzene ring-area units, either $S_{\text {(benzene) }}=+1$ and $C_{\text {(benzene) }}^{1}=+1$ or $S_{\text {(benzene) }}=-1$ and $C_{\text {(benzene) }}^{1}=-1$. Either way, therefore, $S_{\text {(benzene) }} C^{1}$ (benzene) $=1$. Furthermore, for a carbon-carbon bond in benzene, ${ }^{4}$

$$
P_{\text {(benzene) }}=\frac{2}{3} \text { and } \beta \bar{\pi}_{\text {(benzene)(benzene) }}=-\left(\frac{5}{9}\right)
$$

Accordingly, it is clear from expression (15) that, in these units, the ring-current intensity in benzene is $1 / 9$, and this therefore accounts for the factor of 9 when ring currents are expressed as a ratio to the benzene value, as in equation (14).

Tables 1 and 2 give all the necessary data on the quantities $P_{(\mu)}, \beta \bar{\pi}_{(\mu)(\mu)}$, and $\beta \bar{\pi}_{(\mu)(v)}$ (all computed from equations (6), (9), (10) and (13), above) and the $S_{(\mu)}(\mu=$ $1,2, \ldots, 7$, and $v=1,2, \ldots, 7)$, together with knowledge of the set of matrix elements $\left\{C_{(\mu)}^{l}\right\}_{\mu}=1,2, \ldots, 7, i=1,2, \ldots, 7$ needed in order to apply McWeeny's Master Equation (14) to calculate the ring-currents in coronene based on the un-branched spanning-tree illustrated on the righthand side of Figure 3. The data in Tables 1 and 2 are all displayed in single precision - please see later for a discussion on this aspect.

This all results in calculated ring-current intensities (relative to benzene) of $\mathbf{1 . 4 5 9}$ (for the outer rings of coronene) and $\mathbf{1 . 0 3 8}$ (for the inner ring), in satisfactory agreement with the well-established literature values. $^{72,73,22,23}$

(ii) Selection of a Branched Spanning-Tree, Establishment of a Fundamental System of Circuits for that Spanning Tree, and Use of the Gayoso-Boucekkine Unitary-Transformation on the Basis Orbitals. Calculation of Ring Currents

For the conjugated systems most commonly encountered, ${ }^{9}$ to which attempts have been made to apply the McWeeny method, ${ }^{4}$ a continuous (un-branched) spanning-tree can, in general, easily be found, and so there is usually no difficulty in effecting the procedure just

Table 2. Imaginary self- and mutual bond-bond polarisabilities $\left(\beta \bar{\pi}_{(\mu)(\mu)}\right.$ and $\beta \bar{\pi}_{(\mu)(v)}$, respectively) for the seven circuitcompleting bonds/chords of the continuous/un-branched spanning-tree of the coronene molecular-graph depicted on the righthand side of Figure 3

\begin{tabular}{cccccccc}
\hline $\begin{array}{c}\text { Label of circuit- } \\
\text { completing bond }\end{array}$ & (1) & (2) & (3) & (4) & (5) & (6) & (7) \\
\hline (1) & -0.42838 & 0.00913 & 0.00509 & -0.00509 & -0.00913 & -0.05121 & 0.06127 \\
(2) & 0.00913 & -0.43560 & -0.04208 & -0.00405 & -0.01017 & -0.00405 & 0.00913 \\
(3) & 0.00509 & -0.04208 & -0.43560 & -0.04208 & -0.00405 & -0.01017 & 0.00509 \\
(4) & -0.00509 & -0.00405 & -0.04208 & -0.43560 & -0.04208 & -0.00405 & -0.00509 \\
(5) & -0.00913 & -0.01017 & -0.00405 & -0.04208 & -0.43560 & -0.04208 & -0.00913 \\
(6) & -0.05121 & -0.00405 & -0.01017 & -0.00405 & -0.04208 & -0.43560 & -0.05121 \\
(7) & 0.06127 & 0.00913 & 0.00509 & -0.00509 & -0.00913 & -0.05121 & -0.46488 \\
\hline
\end{tabular}




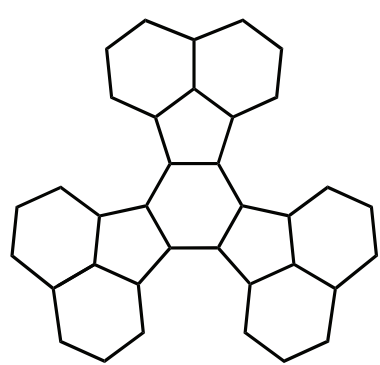

Figure 5. The molecular graph of decacyclene.

described in the previous section. There are, however, certain structures - albeit apparently rare - for which it is not possible to devise an un-branched (continuous) spanning-tree, representing a semi-Hamiltonian path ${ }^{36,66}$ through the molecular graph. To these, the original McWeeny approach, ${ }^{4}$ just described, cannot, without modification, be applied. An example ${ }^{36}$ of such a conjugated system with a non-semi-Hamiltonian moleculargraph is the ten-ring structure decacyclene, illustrated in Figure 5. (It might be observed in passing that establishing the necessary and sufficient conditions for a graph to be Hamiltonian or semi-Hamiltonian is one of the still-unsolved problems in Graph Theory. ${ }^{36,66}$ ) In his original paper, McWeeny did state that '...the result is also valid if the original chain contains branches...' though he did not give any explicit details or examples.

In order explicitly to circumvent this problem, Gayoso and Boucekkine,${ }^{74}$ in a seldom-cited and undeservedly neglected paper, proposed a generalisation of McWeeny's unitary transformation on the basis orbitals ${ }^{4}$ in the presence of the external magnetic-field in such a way that the new transformation enables a ring-current calculation to be based on a branched spanning-tree, such as that illustrated on the right-hand side of<smiles>C1CC2CCC3CCC4CCC5CCC6CCC(C1)C2C6C5C34</smiles><smiles>CCCCCCCC(CCC)CCCCC(C)CCC(C)CC</smiles>

Figure 6. The molecular graph of coronene (left), and (right) a branched spanning-tree of that molecular graph.

Figure 6. This means that not only are structures such as decacyclene (Figure 5) now brought within the scope of the method, but also that, even for structures like coronene - on the left-hand side of Figures 3 and $6-$ for which a continuous (un-branched) spanning-tree is available (such as that on the right-hand side of Figure 3 ), the ring-current calculation could still be based on a branched spanning-tree, if so desired. Thus, if a branched spanning-tree - such as that on the right-hand side of Figure 6 - were adopted for the coronene molecular-graph (illustrated on the left-hand side of Figure 6), the required Fundamental System of Circuits ${ }^{63}$ would be that depicted in Figure 7. These, used in conjunction with the data in Tables 3 and 4 and the 'Master Equation' (equation (14)), again yield ring-current values of ( $c f$. Refs. 72, 73, 22 and 23) of $\mathbf{1 . 4 5 9}$ (for the outer rings of coronene) and $\mathbf{1 . 0 3 8}$ (for the central ring).

The numbers just quoted were computed in double precision, whilst those computed from Figure 4 and presented in Tables 1 and 2 were computed in single precision. A discussion on performing these calculations in single precision and in double precision now follows.<smiles>CCC(C)CCC(C)CCCCC1CCCCCCCCC1CC</smiles><smiles>CCCC(CCCCC(C)CCC(C)CC)C1CCCCC1CC</smiles><smiles>CCCCCc1ccc(C)cc1CCC(C)CCCCC</smiles><smiles>CCCCCCC(CC(C)CC)=C(CCCC)CC(C)CCCC</smiles><smiles>CCCCCCCCC(CCC)CCCCC1CCC(CC)[Te]C1</smiles><smiles>CCCCCCCCC(CCC)C1=C(CC(C)CC)C(C)CCC1</smiles><smiles>CCCCCCCCC1CCCC(CCC(C)CCC(C)CC)C1</smiles>

Figure 7. The Fundamental System of Circuits ${ }^{63}$ associated with the branched spanning-tree (shown on the right-hand side of Figure 6) of the coronene molecular-graph, depicted on the left-hand side of Figure 6. 
Table 3. Coulson bond-orders for the $\mu^{\text {th }}$ circuit-completing bond (middle column) and algebraical areas for the $\mu^{\text {th }}$ fundamental circuit (right-hand column) $(\mu=1,2, \ldots, 7)$ for the branched spanning-tree of the coronene molecular-graph depicted on the right-hand side of Figure 6

\begin{tabular}{ccc}
\hline $\begin{array}{c}\text { Labelling }(\mu) \text { of } \\
\text { fundamental } \\
\text { circuit (see } \\
\text { Figure 7) }\end{array}$ & $\begin{array}{c}\text { Coulson bond- } \\
\text { order of } \\
\mu^{\text {th }} \text { circuit-closing } \\
\text { bond }\end{array}$ & $\begin{array}{c}\text { Signed area of } \\
\mu^{\text {th }} \text { fundamental } \\
\text { circuit (see } \\
\text { Figure 7) }\end{array}$ \\
\hline (1) & 0.5380176262 & 2.00000 \\
(2) & 0.5380176263 & 1.00000 \\
(3) & 0.5380176261 & 3.00000 \\
(4) & 0.5218915077 & -2.00000 \\
(5) & 0.7450420228 & 1.00000 \\
(6) & 0.5218915079 & 1.00000 \\
(7) & 0.5380176261 & 1.00000 \\
\hline
\end{tabular}

(iii) Calculations Effected in Single- or Double Precision.

Table 5 gives the results of calculations of the ringcurrent intensities in the six symmetrically equivalent peripheral-rings of coronene performed in single precision and in double precision, and using both continuous (Figure 3) and branched (Figure 6) spanning-trees as the starting point.

Examination of the results of calculations in single precision (columns 1 and 2 of the main body of Table 5) and in double precision (columns 3 and 4 of Table 5) gives rise to what may superficially appear to be coun- ter-intuitive trends. Namely, when the calculations are performed in single precision seemingly identical results are obtained whether a branched or a continuous (i.e., un-branched) spanning-tree is employed, even though a smaller spread of ring-current values amongst the individual symmetrically equivalent rings is obtained when a branched spanning-tree is chosen. Working in double precision yields both more variation in the average ringcurrent in the six individual peripheral-rings in coronene, as well as a larger standard-deviation. It is an artefact of the method that, when all the contributions to the total ring-current are summed up, some rings are counted more times than others, depending on which starting spanning-tree is chosen. In the example given in Figure 4, the ring bounded by the carbon atoms labelled 5-6-7-8-9-10-5 is counted six times, whereas the ring described by the sequence of carbon atoms $22-23-24-7-6-1-22$ is effectively counted only once. It is for this reason that, wherever possible when performing HLPM calculations, ${ }^{1-4}$ branched spanning-trees should in general be chosen in preference to continuous spanning-trees, as circuit areas in the former tend, on the whole, to be smaller. Since, in equation (14), these circuit areas multiply the bond-orders and the various polarisabilities defined in equations (6)-(13), there is a general liability for round-off errors to be greater if continuous spanning-trees are used. Furthermore, whilst, in the example given here, it may seem preferable to work in single precision, this, too, should be avoided as the reduced precision may yield less variation merely as an artefact of the hardware on which the

Table 4. Imaginary self- and mutual bond-bond polarisabilities $\left(\beta \bar{\pi}_{(\mu)(\mu)}\right.$ and $\beta \bar{\pi}_{(\mu)(v)}$, respectively) for the seven circuitcompleting bonds/chords of the branched spanning-tree of the coronene molecular-graph depicted on the right-hand side of Figure 6

\begin{tabular}{ccccc}
\hline $\begin{array}{c}\text { Label of circuit- } \\
\text { completing bond }\end{array}$ & (1) & (2) & (3) & (4) \\
\hline (1) & -0.4648810239 & -0.0512096967 & 0.0127924144 & 0.0009270662 \\
(2) & -0.0512096967 & -0.4355982327 & 0.0091344912 & 0.0091344911 \\
(3) & 0.0127924144 & 0.0091344912 & -0.4648810240 & 0.0612712542 \\
(4) & 0.0009270662 & 0.0091344911 & 0.0612712542 & -0.4283833234 \\
(5) & 0.0077058376 & 0.0050865769 & 0.0219269056 & 0.0100615576 \\
(6) & 0.0009270662 & -0.0050865769 & 0.0009270661 & 0.0331639966 \\
(7) & 0.0219269058 & -0.0091344912 & 0.0077058376 & -0.0041595107 \\
\hline
\end{tabular}

\begin{tabular}{cccc}
\hline $\begin{array}{c}\text { Label of circuit- } \\
\text { completing bond }\end{array}$ & (5) & (6) & (7) \\
\hline (1) & 0.0077058376 & 0.0009270662 & 0.0219269058 \\
(2) & 0.0050865769 & -0.0050865769 & -0.0091344912 \\
(3) & 0.0219269056 & 0.0009270661 & 0.0077058376 \\
(4) & 0.0100615576 & 0.0331639966 & -0.0041595107 \\
(5) & -0.6719054206 & 0.0100615575 & 0.0127924145 \\
(6) & 0.0100615575 & -0.4283833234 & 0.0100615574 \\
(7) & 0.0127924145 & 0.0100615574 & -0.4648810240 \\
\hline
\end{tabular}


Table 5. Ring-current intensities calculated for the outer rings of coronene carried out in both single precision (columns 1 and 2 of the main body of the Table) and double precision (columns 3 and 4) and based on the continuous spanning-tree depicted on the right-hand side of Figure 3 (row 1) and on the branched spanning-tree depicted on the right-hand side of Figure 6 (row 2)

\begin{tabular}{|c|c|c|c|c|}
\hline & $\begin{array}{l}\text { Average ring-current } \\
\text { intensity expressed to } \\
\text { three decimal-places }\end{array}$ & $\begin{array}{l}\text { Standard } \\
\text { deviation }\end{array}$ & $\begin{array}{l}\text { Average ring-current } \\
\text { intensity expressed to } \\
\text { three decimal-places }\end{array}$ & $\begin{array}{l}\text { Standard } \\
\text { deviation }\end{array}$ \\
\hline & \multicolumn{2}{|c|}{ Calculated in Single Precision } & \multicolumn{2}{|c|}{ Calculated in Double Precision } \\
\hline $\begin{array}{l}\text { Continuous spanning-tree } \\
\text { (Figure } 3 \text { - right-hand side) }\end{array}$ & 1.459 & 0.000429 & 1.463 & 0.002018 \\
\hline $\begin{array}{l}\text { Branched spanning-tree } \\
\text { (Figure } 6-\text { right-hand side) }\end{array}$ & 1.459 & 0.000121 & 1.459 & 0.000172 \\
\hline
\end{tabular}

calculations are being performed. Double precision should, therefore, be used at all times. This issue becomes more pertinent when investigating systems with large rings or with large numbers of rings (as we shall do later in this paper).

\section{APPLICATION TO 'SUPER-RING' SYSTEMS IN ORDER TO ASSESS THE AWA RULE}

This HLPM approach, ${ }^{1-4}$ as described here in detail, has been applied to the seven super-ring structures considered by Aihara in Ref. 27 and illustrated in Figure 2. In view of the considerations of the previous section, the calculation of all the ring currents and bond currents reported here has, in each case, (a) been based on a branched spanning-tree and (b) has been carried out in double precision. The results for Aihara Structures 2, 4, 5 and $\mathbf{6}$ (of Figure 2) are displayed in Figure 8. Corresponding results for single precision calculations based on continuous spanning-trees in structures $\mathbf{1 ,} \mathbf{3}$ and $\mathbf{7}$ are already available in Ref. 22.

Ring-current intensities are written (in black) in the centre of each ring; when such ring currents are positive (that is, diamagnetic), they are considered to be circulating in the anti-clockwise sense around the ring in question: when negative (paramagnetic), they are regarded as flowing in the clockwise direction around that ring. The corresponding bond-currents are written (in red, in the on-line version of the paper) along the respective bonds, and they flow in the direction indicated by the arrow on each bond. As the present authors have pointed out previously, ${ }^{48,22,23}$ bond currents and ring currents are not independent quantities because the bond currents can be deduced from the ring currents - as, indeed, we do here - by (algebraical) addition ${ }^{48}$ by virtue of the fact that, at the microscopic, molecular level, the system of currents respects Kirchhoff's Law of Current Conservation at junctions in macroscopic,

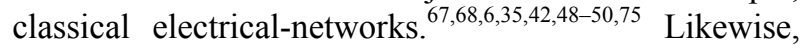
the ring currents can be reconstituted from a complete set of bond currents.

As is implied from equation (14), the intensities both of bond currents and ring currents are expressed as a ratio to the corresponding currents calculated, by the same method, for benzene; they are thus dimensionless quantities. It is emphasised again that, in this topological model, all Coulomb integrals, ${ }^{1} \alpha$, have been assumed to be the same as that for a carbon atom in benzene, and all resonance integrals, ${ }^{1} \beta$, are set equal to the standard value appropriate ${ }^{1}$ for a carbon-carbon bond in benzene.

An aspect of the prescription for evaluating strictly 'topological' ring-currents ${ }^{9-11}$ is that the areas of $[N]$-membered rings in conjugated structures are conventionally estimated from the formula that applies to regular polygons of the same side length:

$$
\left(\begin{array}{c}
\text { Area of a Regular Polygon } \\
\text { Area of a Regular Hexagon }
\end{array}\right)=\left(\frac{N \cot \left(\frac{\pi}{N}\right)}{6 \cot \left(\frac{\pi}{6}\right)}\right)
$$

This is tantamount to invoking the simplification of regarding all rings as being regular polygons of the same side length. At least from a purely geometrical point of view, this assumption is precisely correct for the four, six and twelve-membered rings of Aihara structure 7 (anti-kekulene), illustrated in Figure 2 and discussed in Ref. 22, because all three types of rings in that structure tessellate exactly, in the plane, as regular polygons. The same is true for all of the geometrically planar benzenoid structures dealt with in Ref. 9. However, when considering some of these super-ring structures, ${ }^{26,27}$ such as the benzenoid hydrocarbons 1, 2, 5 and $\mathbf{6}$ (Figure 2) - whose central ring is, geometrically speaking, effectively a 'hole' in an otherwise perfectly tessellated hexagonal-lattice - it seems logical to estimate the area of this central ring as being the total area of the 'missing' hexagons. We therefore take these central-ring areas to be seven benzene-hexagon units in the case of 1 , six benzene-hexagon units in the case of $\mathbf{2}$, and four- and three benzene-hexagon units for $\mathbf{5}$ and $\mathbf{6}$, respectively. Structure $\mathbf{4}$ is not really a super-ring ${ }^{26,27}$ structure at all, as six of its bonds may be thought of as forming a part of both the 'outer perimeter' and the 
'inner ring'; nevertheless, it was included in Aihara's list and, consequently, it is considered here, the area of its central ring being taken to be that of seven benzene hexagons. Assumptions made about the areas of the central rings of structures 3 and 7 are discussed in detail in Ref. 22. Very early studies ${ }^{76,77}$ connected with the HLPM method showed that, in any case, its conclusions are often remarkably insensitive to the actual details of the assumptions made about ring areas. A related point was very recently made by Fowler and Myrvold in Ref. 44, and by ourselves in Ref. 12.

It should be noted that some of the structures 1-7 might possibly be non-planar - either as a result of proton-proton steric interactions and/or because of angle strain arising from too large a deviation from the idealized $\mathrm{sp}^{2}$-hybridised $120^{\circ} \mathrm{C}-\mathrm{C}-\mathrm{C}$ angles. As previously emphasised, ${ }^{9-11,22,23}$ the HLPM topological ring-currents calculated for any conjugated systems dealt with here that might be non-planar are, in effect, those for a hypothetical structure having the same carboncarbon connectivity as the actual molecule under consideration but envisaged as if it were geometrically planar.

Finally, from the ring currents reported in Figure 8 it is possible to deduce the quantities $\left(\chi_{\perp}{ }^{\pi \text {-London }}\right.$ (structure) $/ \chi_{\perp}{ }^{\pi \text {-London }}($ benzene) $)$, given by the expression $^{1-6,23,43}$

$$
\left(\frac{\chi_{\perp}^{\pi-\text { London }}(\text { structure })}{\chi_{\perp}^{\pi-\text { London }}(\text { benzene) })}\right)=\sum_{i=1}^{r}\left(\frac{J_{i}}{J_{\text {benzene }}}\right) \times\left(\frac{s_{i}}{s_{\text {benzene }}}\right)
$$

where

(a) $\chi_{\perp}{ }^{\pi \text {-London }}$ (structure) and $\chi_{\perp}{ }^{\pi \text {-London }}($ benzene) are the calculated 'London' contributions to the $\pi$-electron magnetic-susceptibility, ${ }^{1-6,43,23}$ perpendicular to the (assumed) molecular plane, of the conjugated structure in question, and of benzene (respectively),

(b) $\left(J_{i} / J_{\text {benzene }}\right)$ is defined in equation (14), and

(c) $\left(s_{i} / s_{\text {benzene }}\right)$ is the area of the $i^{\text {th }}$ ring (expressed as a multiple of the area of a standard benzene-hexagon) of the conjugated structure in question (which, in all, has $r$ rings).

The summation on the right-hand side in equation (17) runs up to $r$, the number of rings in the system under study. As mentioned, these (idealised) ring-areas were estimated from equation (16) in the case of structures $\mathbf{3}$ and $\mathbf{7}$ and, in the case of the central rings of $\mathbf{1}, \mathbf{2}$, $\mathbf{4}, \mathbf{5}$, and $\mathbf{6}$, by counting the number of hexagons that would fill the 'hole' in the otherwise completely tessellated hexagonal-network. The resulting 'London' susceptibility-ratios for 1-7 are presented in the right-hand column of Table 6 .

\section{DISCUSSION}

Ring-current and bond-current data on the seven superring structures (1-7) studied by Aihara ${ }^{27}$ (Figure 2) are quantitatively presented in Figure 8 for systems 2, 4, 5 and 6 and in Figure 2 of Ref. 22 for 1, 3, and 7 (but please see footnotes (e), (f) and (g) of Table 6 for the quantitative influence that using single precision or double precision and a continuous spanning-tree or a branched spanning-tree has on the numerical values of calculated ring-currents). Each of the structures 1, $\mathbf{2}, \mathbf{3}$ and $\mathbf{5}$ has a [ $4 m+2]$ carbon-atom periphery and a $[4 n+2]$-membered central-ring; 6 and 7 have $[4 m]$ carbon-atom peripheries and $[4 n]$-membered centralrings. (Hexa- $m$-phenylene (4) is a somewhat special case, and it will separately be considered, later). It may be noted from the right-hand column of Table 6 that the overall 'London' magnetic susceptibilities ${ }^{1-6,23,43}$ of 1-7 are, overall, more-or-less strongly diamagnetic, with the notable exception of the aptly named anti-kekulene (7), which exhibits an overall paramagnetic susceptibility of size more than 13 times that of the diamagnetic susceptibility calculated for benzene.

It can be seen from Table 6 that all structures with $[4 m+2]$ peripheries and $[4 n+2]$ central-rings ( $1-3$ and 5) do indeed bear diamagnetic ring-currents in their peripheral rings and a diamagnetic one in their central rings. On first (but superficial) consideration, therefore, the $\pi$-electron magnetic properties of these structures seem intuitively to be in accord with the AWA model. However, when the data are examined in more detail it is seen that the intensity of the diamagnetic ring-current in the central ring is, in every case, smaller than the diamagnetic ring-current intensity in any of the outer, peripheral rings. The consequence of this is that, for every single one of the bonds in the central rings of these four structures, the net flow of current is in the clockwise (that is, paramagnetic) direction around that central ring, as a result of this competition between the diamagnetic current in the outer rings and the (smaller) diamagnetic ring-current in the central rings. Despite initial intuition, therefore, it should properly be concluded that the AWA model is - after all — violated by structures 1-3 and 5, all of which have [ $4 m+2]$ peripheries and $[4 n+2]$-membered central-rings.

We next consider the analogous situation in the case of the two structures considered ([9]-coronaphene (6) and anti-kekulene (7)) that have a $[4 m]$ periphery and a $[4 n]$ central-ring. The present authors have already noted ${ }^{22}$ that the AWA rule does not hold in the case of 7 - at least, when judged by calculations based on this HLPM formalism. ${ }^{1-4,9-13}$ The peripheral rings of 7 do bear avowedly paramagnetic topological $\pi$-electron ring-currents $(-1.656$ for the four-membered rings on 
the perimeter and -0.606 for the peripheral sixmembered rings ${ }^{22}$ — please see footnote (g) in Table 6); the peripheral bonds in these rings are (by definition) unshared with any other rings and thus carry paramagnetic bond-currents - as would be expected from the AWA model. The central ring also supports a strong paramagnetic ring-current $(-1.386$ - please see footnote $(\mathrm{g})$ of Table 6$)$. However, since the magnitude of the paramagnetic ring-current intensity in the central ring is smaller than that of the paramagnetic ring-current sustained by the six-membered rings on the periphery, the end result is that the bond currents along those bonds shared between the central ring and the sixmembered rings on the periphery are diamagnetic (that is, are anti-clockwise) in nature when considered from the point of view of the central ring. ${ }^{22}$ As was argued in Ref. 22, for those bonds shared between the (18membered) central-ring and the four-membered rings on the perimeter, exactly the opposite is the case and these bonds bear currents flowing in the paramagnetic (that is, clockwise) direction in the central ring. Half of the bonds in the central ring are thus predicted ${ }^{22}$ to bear topological $\pi$-electron currents that, from the point of view of the central ring, are diamagnetic, and the other half of the bonds in that central ring are predicted to bear paramagnetic currents. Accordingly, in Ref. 22, and in the fifth column of Table 6 , here, the topological bond-currents in the central ring of 7 are described as 'mixed'; structure 7, therefore, likewise does not obey the strict provisions of the AWA rule. ${ }^{14-24}$

By contrast, we present new results (in Figure 8 and Table 6) for another structure ([9]-coronaphene (6)) which, like anti-kekulene $(7)$, has a $[4 m]$ carbon-atom periphery and also a $[4 n]$-membered central-ring. This is seen (Figure 8 and Table 6) to exhibit diamagnetic ringcurrents in the peripheral rings and a paramagnetic ringcurrent in the central ring. Unlike in 7, the algebraical competition for direction of current-flow in the central ring of [9]-coronaphene (6) determines that currents in all bonds in the central ring flow in the paramagnetic

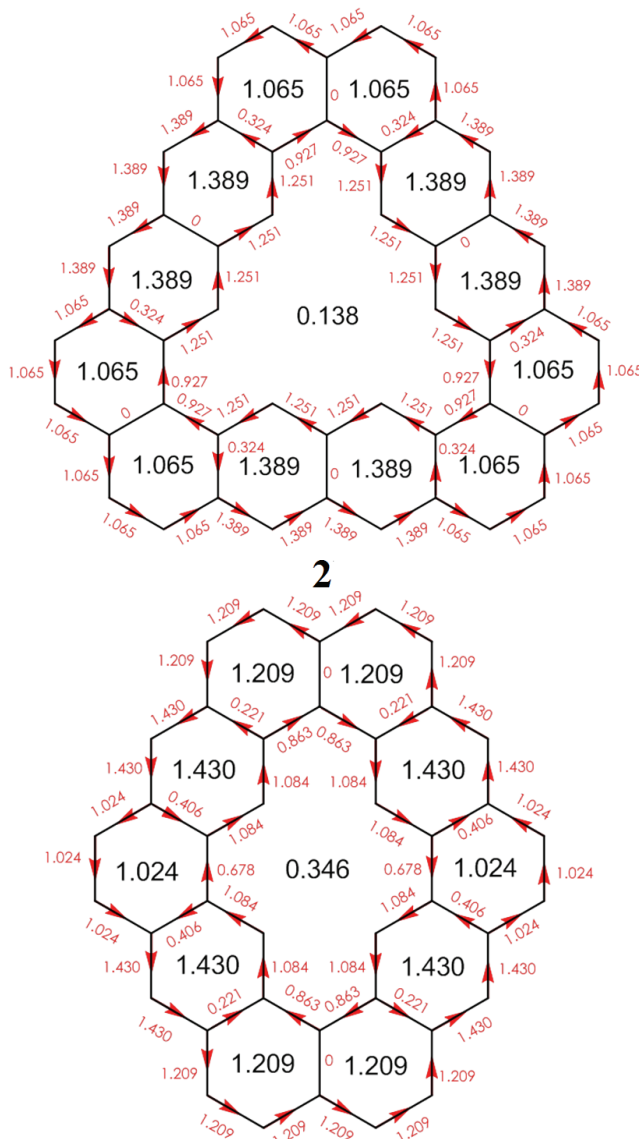

5

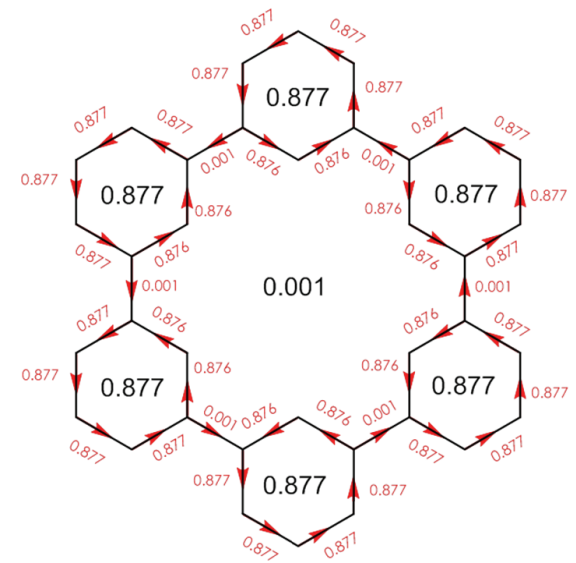

4

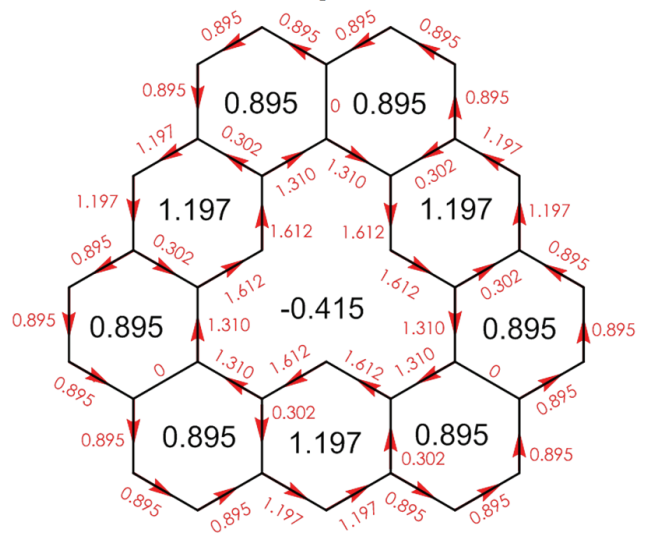

6

Figure 8. Ring currents (in black) and the associated bond-currents (in red, in the on-line edition) in structures 2, 4, 5 and $\mathbf{6}$ calculated by the $\mathrm{HLPM}^{1-4}$ approach, ${ }^{9-11}$ as described in the text. The ring currents and bond currents are dimensionless quantities; accordingly, all are appropriately depicted here as pure numbers, without units. Positive (diamagnetic) ring-currents are considered to circulate anti-clockwise around their respective rings whilst negative (paramagnetic) ring-currents flow in the clockwise sense around those rings. The numerous bond-currents flow in the direction indicated by the arrow pointing along each bond. 
Table 6. Qualitative summary of the HLPM calculations on structures 1-7 and quantitative assessment of their overall 'London' $\pi$-electron magnetic-susceptibilities

\begin{tabular}{|c|c|c|c|c|c|c|}
\hline $\begin{array}{c}\text { Structure } \\
\text { (labelled and } \\
\text { displayed in } \\
\text { Figure 2) }\end{array}$ & $\begin{array}{l}\text { Number of } \\
\text { carbon } \\
\text { atoms in } \\
\text { the outer } \\
\text { periphery }\end{array}$ & $\begin{array}{c}\text { Number of } \\
\text { carbon atoms } \\
\text { in the central } \\
\text { ring }\end{array}$ & $\begin{array}{l}\text { Nature of the } \\
\text { individual bond- } \\
\text { currents for } \\
\text { bonds on the } \\
\text { outer periphery } \\
\end{array}$ & $\begin{array}{l}\text { Nature of the } \\
\text { individual bond- } \\
\text { currents for bonds } \\
\text { in the central ring }\end{array}$ & $\begin{array}{l}\text { Is this } \\
\text { compliant } \\
\text { with the } \\
\text { AWA } \\
\text { rule? }\end{array}$ & $\begin{array}{l}\text { 'London' } \pi \text {-Electron } \\
\text { magnetic- } \\
\text { susceptibility ex- } \\
\text { pressed as a ratio to } \\
\text { the benzene value }{ }^{(b),(c)} \\
\end{array}$ \\
\hline $\begin{array}{c}\text { Kekulene }^{(\text {a) }} \\
\text { (1) } \\
\text { Triangular- }\end{array}$ & $\begin{array}{c}30 \\
(4 m+2)\end{array}$ & $\begin{array}{c}18 \\
(4 n+2)\end{array}$ & Diamagnetic & Paramagnetic & NO & $15.5^{(\mathrm{d}),(\mathrm{e})}$ \\
\hline $\begin{array}{l}\text { Triangular- } \\
\text { kekulene } \\
\text { (2) }\end{array}$ & $(4 m+2)$ & $\begin{array}{c}18 \\
(4 n+2)\end{array}$ & Diamagnetic & Paramagnetic & NO & 15.6 \\
\hline $\begin{array}{l}\text { Azulenoid- } \\
\text { kekulene }^{(\text {a) }} \\
\text { (3) }\end{array}$ & $\begin{array}{c}30 \\
(4 m+2)\end{array}$ & $\begin{array}{c}18 \\
(4 n+2)\end{array}$ & Diamagnetic & Paramagnetic & NO & $18.6^{(\mathrm{f})}$ \\
\hline $\begin{array}{l}\text { Hexa-m- } \\
\text { phenylene } \\
(\mathbf{4})\end{array}$ & $\begin{array}{c}30 \\
(4 m+2)\end{array}$ & $\begin{array}{c}18 \\
(4 n+2)\end{array}$ & $\begin{array}{l}\text { Diamagnetic and } \\
\text { (virtually) zero }\end{array}$ & $\begin{array}{l}\text { Paramagnetic and } \\
\text { (virtually) zero }\end{array}$ & NO & 5.3 \\
\hline $\begin{array}{c}{[10]-} \\
\text { Coronaphene } \\
(\mathbf{5})\end{array}$ & $\begin{array}{c}26 \\
(4 m+2)\end{array}$ & $\begin{array}{c}14 \\
(4 n+2)\end{array}$ & Diamagnetic & Paramagnetic & NO & 14.0 \\
\hline $\begin{array}{c}{[9]-} \\
\text { Coronaphene } \\
(6)\end{array}$ & $\begin{array}{c}24 \\
(4 m)\end{array}$ & $\begin{array}{c}12 \\
(4 n)\end{array}$ & Diamagnetic & Paramagnetic & NO & 7.7 \\
\hline $\begin{array}{c}\text { Anti- } \\
\text { kekulene }^{(a)} \\
\text { (7) }\end{array}$ & $\begin{array}{c}24 \\
(4 m)\end{array}$ & $\begin{array}{c}12 \\
(4 n)\end{array}$ & Paramagnetic & $\begin{array}{l}\text { Mixed (Overall } \\
\text { Average: Para- } \\
\text { magnetic) }\end{array}$ & NO & $-13.4^{(\mathrm{g})}$ \\
\hline
\end{tabular}

(a) Data for kekulene (1), azulenoid-kekulene (3), and anti-kekulene (7) were initially given in Table 1 of Ref. 22. (Please also see footnotes (d), (f) and (g) to this Table.)

(b) This is the quantity $\left(\chi_{\perp}{ }^{\pi \text {-London }}\right.$ (structure) $/ \chi_{\perp}{ }^{\pi-\text { London }}$ (benzene)) calculated from equation (17), with topological ring-currents, $\left(J_{\mathrm{i}} / J_{\text {benzene }}\right)$, deduced from equation (14) and relative ring-areas, $\left(s_{\mathrm{i}} / s_{\text {benzene }}\right)$, evaluated via equation (16) (for 3 and 7$)$ or (in the case of structures $1,2,4,5$ and $\mathbf{6})$ according to the procedures described in the paragraph following equation (16), in the text.

(c) A positive value (found for structures 1-6) indicates overall diamagnetic 'London' susceptibility: a negative value (as in the sole case of anti-kekulene (7)) indicates an overall paramagnetic 'London' susceptibility (all in a direction at right angles to their assumed molecular-planes).

(d) It might be observed in passing that kekulene (1) could be thought of as being the likewise hexagonally symmetric dodecabenzocoronene $\left(\mathrm{C}_{42} \mathrm{H}_{18}\right.$, the last structure in Table XIII - page 244 - of Ref. 9) but with seven symmetrically configured interior-rings excised. The HLPM ring-currents appropriate for this latter structure were published by Maddox and McWeeny, some fifty years ago. ${ }^{72}$ When these are used, in conjunction with equation (17), to estimate the London diamagnetic-susceptibility for the 19-ring structure $\mathrm{C}_{42} \mathrm{H}_{18}$, a value of about 35 times the benzene value is obtained - twice that of kekulene (1), even though dodecabenzocoronene has only $50 \%$ more rings than does kekulene.

(e) Kekulene (1). The same value of this susceptibility ratio (to one decimal-place) was estimated from ring currents reported in Ref. 22 that were calculated in single precision and based on a continuous spanning-tree. With the several rings as depicted in structure 4 in Figure 2 of Ref. 22, the ring-current intensities calculated in double precision and based on a branched spanning-tree (in bold), used here, compared with those (in brackets) in single precision and based on an un-branched spanning-tree (from Ref. 22), are as follows. For the six-membered rings on the perimeter: 1.359 (1.360) and 0.998 (0.999); and for the central ring: 0.190 (0.191).

(f) Azulenoid-kekulene (3). The value 18.3 (to one decimal-place) was estimated for this susceptibility ratio from ring currents reported in Ref. 22 that were calculated in single precision and based on an un-branched spanning-tree, which are all slightly smaller than those (employed here) that are calculated in double precision and are based on a branched spanning-tree. With the various rings as depicted in structure 5 in Figure 2 of Ref. 22, the ring-current intensities calculated in double precision starting with a branched spanning-tree (in bold), used here, compared with those (in brackets) in single precision based on a continuous spanning-tree (from Ref. 22), are as follows. For the pentagonal rings on the perimeter: 1.066 (1.060); for the heptagonal rings on the perimeter: 1.286 (1.279); and for the eighteen-membered central-ring: $\mathbf{0 . 3 6 0}(0.345)$.

(g) Anti-kekulene (7). The value -13.5 (to one decimal-place) was estimated from the (paramagnetic) ring-currents reported in Ref. 22 , which were calculated in single precision and based on a continuous spanning-tree, and which were all marginally larger in magnitude than the paramagnetic ring-currents calculated in double precision and based on a branched spanning-tree (used here). With the several rings as depicted in structure 6 in Figure 2 of Ref. 22, the ring-current intensities (in bold) calculated in double precision and based on a branched spanning-tree, compared with those (in brackets, from Ref. 22) in single precision based on an un-branched spanning-tree, are as follows. For the peripheral six-membered rings: $\mathbf{- 0 . 6 0 6}(-0.609)$; for the four-membered rings that lie on the periphery: $\mathbf{- 1 . 6 5 6}(-1.660)$; and for the twelve-sided central-ring: $\mathbf{- 1 . 3 8 6}(-1.393)$. 
sense (that is, clockwise). This much would be expected if the AWA rule held. However, in the case of $\mathbf{6}$, compliance with the AWA model fails because of the outer, peripheral rings. Despite the 24-carbon-atom perimeter $([4 m]$, with $m=6)$, all nine rings on that perimeter bear $\pi$-electron ring-currents that are unambiguously diamagnetic. The consequence of this observation is that the unshared bonds that form the periphery of 6 support diamagnetic $\pi$-electron bond-currents. Accordingly, although the central ring of $\mathbf{6}$ behaves magnetically as would be anticipated if the AWA model were respected, the peripheral rings do not.

Hexa- $m$-phenylene (4) was included in Aihara's list $^{27}$ of super-ring ${ }^{26,27}$ molecules (all depicted in Figure 2) but it is fundamentally different from the other six structures so far considered here. Six of its bonds, which are all formally single and effectively carry zero ringcurrent, may be considered to form a part of both the inner ring and the outer ring. As can be seen from Figure 8, Hexa- $m$-phenylene (4) essentially consists (from a ring-current point of view) of six, completely isolated benzenoid rings (each bearing a ring current of intensity just less than $90 \%$ of the benzene value) and an 18-membered inner-ring supporting (to three decimal-places) zero ring-current, according to the singleprecision calculation, and a negligible 0.001 , according to the calculation carried out in double precision, the results of which are shown in Figure 8. (It should be pointed out that hexa- $m$-phenylene is one of those structures, like decacyclene depicted in Figure 5, for which a continuous spanning-tree cannot be drawn; accordingly, both the single-precision and the double-precision calculations being discussed here were based on a branched spanning-tree.) In order to investigate whether this 0.001 was just a 'round-off' error for what is in fact precisely zero, the latter calculation was checked by means of repeat calculations based on three different branched spanning-trees; each of these computations, when rounded to three decimal places, gave rise to ringcurrent values of 0.877 for the six-membered rings and verified the value of 0.001 for the central ring, as depicted in structure 4 of Figure 8.

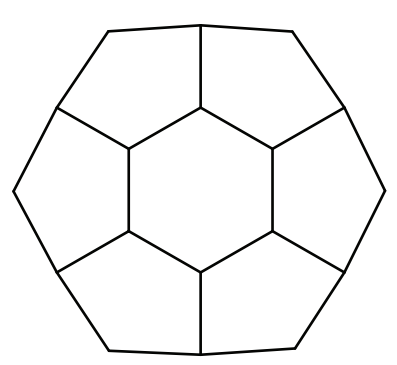

8

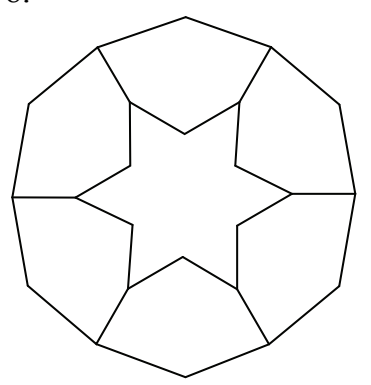

9
Figure 9. Two super-ring systems, $\mathrm{C}_{18} \mathrm{H}_{6}(\mathbf{8})$ and $\mathrm{C}_{24} \mathrm{H}_{6}(\mathbf{9})$, each having precisely four Kekulé structures, discussed by Agranat et al. ${ }^{78}$
The outer ring $([4 m+2], m=7)$ is seen (Figure 8, structure 4) to exhibit diamagnetic bond-currents (of magnitude $c a$. 0.88 ) in 24 of its bonds, and virtually no current at all in the remaining six of its bonds. The central ring is likewise $[4 n+2]$-membered (with, here, $n=4$ ). Its essentially zero ring-current does virtually nothing to oppose the current of $c a$. 0.88 extant in the bonds that it shares with the six-membered rings. Hence, this central, 18-membered ring bears clockwise (that is, paramagnetic) currents in 12 of its bonds, and what may be considered to be virtually zero current in its remaining six bonds.

Thus, even on the somewhat limited interpretation of hexa- $m$-phenylene (4) as a 'super-ring' molecule ${ }^{26,27}$ that has been entertained here, this structure also violates the AWA model.

It is seen, therefore, that none of the seven superring structures of Figure 2, discussed by Aihara in Ref. 27, obeys the AWA 'rule'.

As a postscript, it would perhaps be useful to examine two carefully 'designed' structures — an adjective deliberately invoked by Monaco et al. in Ref. 20 mentioned many years ago by Agranat, Hess and Schaad, ${ }^{78}$ which, according to our arguments recently outlined in Refs. 12 and 13, are prima facie cases for respecting the AWA rule - but see also the very recent Ref. 24, for a contrary view based on ipso-centric $a b$ initio calculations. ${ }^{17-20,32,33}$ These arguments followed the lead started by Fowler et al., ${ }^{17-21}$ that such structures are more likely to obey the AWA 'rule' if the outer periphery and the inner ring are what Fowler et al. call 'decoupled'. ${ }^{17-21}$ This means that the transverse $^{25} /$ spokes $^{21}$ bonds (Figure 1) that connect the outer perimeter to the inner ring never appear as anything other than single bonds in any Kekulé structure that can be devised for the conjugated system as a whole. Another way of stating this is to say that the Pauling bond$\operatorname{order}^{79,80}$ of such bonds must be zero. Yet another equivalent way of saying this is to remark that superring structures of this type have precisely four Kekulé structures (such as are displayed, for example, for the structure 7-coronene in Figure 2 of Ref. 12.) The structures in question, $\mathrm{C}_{18} \mathrm{H}_{6}$ and $\mathrm{C}_{24} \mathrm{H}_{6}$ (labelled 8 and 9, respectively, in Figure 9), were described by Agranat et $a l .^{78}$ (implicitly in the case of $\mathbf{8}$, and explicitly so in the case of 9) as having only four Kekulé structures.

These networks have therefore been subjected to an HLPM topological calculation as described in the first half of this paper. The computation for structure $\mathbf{8}$ proceeded routinely, and the results are shown in the ring-current and bond-current maps of Figure 10. With structure 9, however, there was a difficulty: the eigenvectors were obtained as usual, and the eigenvalues displayed the expected 'pairing' around zero — as is appropriate for a bipartite graph/alternant hydrocarbon 


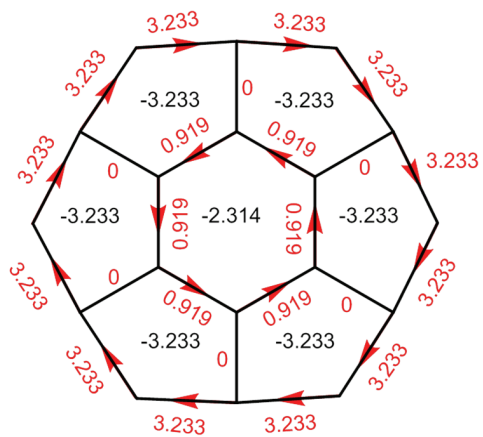

Figure 10. Ring-current and bond-current maps for structure 8, with a six-membered central ring $([4 n+2]$, with $n=1)$ and 12-membered periphery ( $[4 m]$, with $m=3$ ). For conventions on ring currents (black) and bond currents (red, in the on-line version of the paper) please see the caption to Figure 8.

as a result of the Coulson-Rushbrooke Theorem ${ }^{1,81}$ but application of the Aufbau process ${ }^{41,58-60}$ yielded a triplet ground-state. This meant, therefore, that the required MO quantities - the Coulson bond-orders (equation (6)), and self- and mutual imaginary bondbond polarisabi-lities (equations (9), (10) and (13)) could not be evaluated. As a result, ring-current intensities by the HLPM approach were not accessible for this structure 9. (An analogous problem was encountered in Ref. 13 when trying to compute the topological ringcurrents in the first member of the $[r, s]$-coronene series, ${ }^{25,13}[3,12]$-coronene.)

Nevertheless, as indicated by the bond-current map in Figure 10, the symmetrical, decoupled structure 8 does indeed respect the AWA rule, as anticipated, when judged by the HLPM ${ }^{1-4}$ 'topological' ${ }^{\text {9-13 }}$ approach, in that the current around the (unshared) bonds that constitute the 12-membered perimeter is in the clockwise (paramagnetic) direction and that around the (shared) bonds of the six-membered central-ring is in the anti-clockwise (diamagnetic) sense. This former arises because the five-membered rings, each contributing two unshared bonds to the perimeter, themselves actually support paramagnetic ring-currents; the diamagnetic circulation around the central ring arises not because that ring actually bears a diamagnetic ringcurrent per se, but because it bears a paramagnetic ringcurrent which is however smaller in magnitude than the paramagnetic ring-current carried by the peripheral fivemembered rings with each of which it shares a bond. The paramagnetic ring-current (of magnitude 2.314) that the six-membered ring drives around the shared bonds in its own ring is, accordingly, swamped by the larger current (of size 3.233) that is being driven through those bonds in the opposite direction by the peripheral five-membered rings, each sharing an edge with the central ring. The net result of this internal competition in the shared bonds that constitute the six-membered central-ring is that current flows in the anti-clockwise (diamagnetic) direction around that ring. Hence, the HLPM ${ }^{1-4}$ 'topological' ring-currents indicate that the AWA rule is complied with, in the case of structure 8.

\section{CONCLUSIONS}

Superficially — judged merely on the basis of their structural formulae - the Aihara super-ring structures of Figure 2 would appear to have been viable candidates for conjugated systems respecting the AWA rule. However, the present authors have recently argued ${ }^{12,13}$ that such structures are most likely to obey the AWA 'rule' if the outer periphery and the inner ring are decoupled (but see also Ref. 24). It can be shown by actually attempting to draw Kekulé structures and thus creating practical counter-examples that none of these conjugated systems (1-7) is decoupled, in this sense; furthermore, as we have demonstrated here, none of them (at least, according to our HLPM ${ }^{1-4}$ calculations) obeys the AWA 'rule'. Structure 8 (Figure 9), on the other hand, is decoupled (and, incidentally, it has symmetrically equivalent peripheral-rings) and, as its HLPM $^{1-4}$ 'topological ${ }^{\text {9-13 }}$ current-map displayed in Figure 10 confirms, it does respect the AWA model, when judged according to the calculations based on this formalism.

It is therefore once again concluded that

(a) most coupled super-ring structures that have been tested by the HLPM approach seem not to obey the AWA 'rule';

(b) those super-ring systems that are decoupled seem to be more likely candidates for respecting the AWA model, when tested by HLPM ring-current and bond-current calculations.

Statement (a), above, is verified by our calculations on the Aihara ${ }^{27}$ Structures 1-7, studied here, as well as by all the calculations reported in Refs. 22 and 23. Statement (b) is verified by our calculations on structure $\mathbf{8}$, here, and by those reported on the recentlydefined $p$-coronenes in Ref. 12 - but see also the very recent Ref. 24 for a contrary view - and the longknown $[r, s]$-coronenes, considered in Ref. 13.

We must, however, caution that exceptions have been noted to both of the above; they are especially to be found amongst the eighteen isomers of coronene, initially studied by Balaban, Bean and Fowler ${ }^{21}$ by their $a b$-initio, 'pseudo- $\pi$ ' approach, ${ }^{34}$ and later tested by the present authors using the topological ${ }^{9-13}$ HLPM $^{1-4}$ process described in this paper; the same structures have also since been studied by Randić et al. ${ }^{82}$ by application of their method involving circuits of conjugation. ${ }^{83,84,44,48}$ Each of these eighteen structures is a [6]annulene-within-an-[18]-annulene super-ring structure - i.e. they are all ' $[4 n+2]$-annulenes-within- $[4 m+2]$ - 
annulenes'. All topological ring-current intensities in all $(7 \times 18=126)$ rings in these eighteen structures are diamagnetic, ${ }^{23}$ and so the AWA rule is obeyed in these structures only when the diamagnetic topological ringcurrent intensity in the central ring is larger than all of the diamagnetic ring-currents in the peripheral rings; (see, for example, Figure 3 of Ref. 22 and the discussion therein concerning coronene itself.) The exceptions found so far (based on the HLPM tests reported in Figure 1 of Ref. 23) are as follows:

(a) Structures (numbered as in Refs. 21 and 23) that, despite being coupled (that is, having more than four Kekule structures), appear to conform to the AWA model: structures 5 (with 7 Kekulé structures), ${ }^{21} \mathbf{6}$ (with 8 Kekulé structures), ${ }^{21} 7$ (with 9 Kekulé structures), ${ }^{21} \mathbf{8}$

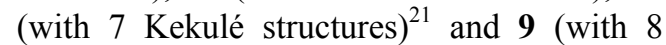
Kekulé structures). ${ }^{21}$

(b) One structure that is decoupled but, nevertheless, does not appear to respect the provisions of the AWA 'rule' when tested by the HLPM topological approach - or, indeed, by the $a b$ initio 'pseudo- $\pi$ ' formalism ${ }^{21,34}$ (though this is contradicted $^{82}$ when the Randić 'conjugationcircuit $^{83,84}$ approach is applied): structure $\mathbf{1 8}$ (with 4 Kekulé structures). ${ }^{21}$

The overall statistics concerning this sample of eighteen isomers of coronene $e^{21,23,82}$ when treated by the HLPM approach may thus be summarised as follows: $(i)$ of 15 coupled structures (1-15 of Refs. 21 and 23), five (listed above) obey the AWA rule (with a ratio, therefore, of $1: 2$, for structures in this set that respect the rule), and (ii) of three decoupled structures (16-18 of Refs. 21 and 23), two (16 and 17) obey the AWA rule (with, a correspondingly reversed ratio of $2: 1$ of structures of this type that respect the AWA rule).

It is seen, therefore, the AWA rule is by no means universal. All that can properly be conjectured from the data considered here is that there appears to be a greater probability of compliance with the AWA rule in decoupled super-ring structures than in coupled ones.

It may, however, be noted finally that all of the decoupled structures so far examined by the HLPM ${ }^{1-4}$ approach that do, in addition, possess symmetrically equivalent peripheral-rings (such as the $p$-coronenes discussed in Ref. 12 and structure 8 considered here (Figure 10)), obey the AWA rule; and yet neither of the two (likewise symmetrical) de-coupled $[r, s]$-coronenes - $[4,8]$-coronene and $[6,6]$-coronene (coronene itself) ${ }^{13}$ - is compliant with the AWA rule. However, it should be pointed out that very recent calculations ${ }^{24}$ on the $p$ coronenes $^{12}$ by the ipso-centric ab-initio method ${ }^{17-20,32,33}$ have disagreed with those ${ }^{12}$ based on the rudimentary graph-theoretical HLPM formalism ${ }^{1-4,9-11}$ being exploited here.
The HLPM topological approach has had considerable exposure in the literature during the last five or six years. ${ }^{9-13,22,23,48}$ Because of this, in addition to outlining our conclusions above concerning the applicability of the AWA rule to super-ring structures, we have taken the opportunity, in this analysis, to use these calculations - on the seven Aihara ${ }^{27}$ super-ring structures (1-7 of Figure 1), and one of the decoupled structures ( 8 of Figure 9) discussed by Agranat et al. ${ }^{78}$ — as a vehicle for presenting step-by-step practical details of how actually to carry out an HLPM $^{1-4}$ topological $^{9-11}$ ringcurrent calculation on a given conjugated system. The complete intermediate stages of such a computation set out in such a way that any interested reader could, if desired, perform such calculations for himself - have not, to our knowledge, previously been conveniently presented, as they are here, all in one place.

We are motivated to do all this because of our belief that, as Haigh and one of the present authors asserted many years ago, ${ }^{5}$ the rudimentary ring-current picture is "...so pictorial that one can almost feel what is happening when a [conjugated] molecule is subjected to a magnetic field' and that, as they put it, '...this is one of its inherent strengths.' Furthermore, as the present authors have recently emphasised, ${ }^{12}$ certain moresophisticated approaches, despite their being 'ab initio', still require prescriptions for specific wave-functions and possibly other assumptions and parameterisations. Accordingly, Gomes and one of us, in Ref. 8, were of the opinion that '...the semi-empirical approaches still have something to contribute, at least conceptually.' Those authors observed ${ }^{8}$ that, although the ab-initio formulations are of course potentially of considerable value in making accurate predictions of experimentally verifiable quantities, the problem with them is that '...the more complex they are the more difficult they become to interpret and visualise.' More-simplistic and intuitive approaches, by contrast, frequently encapsulate what Coulson ${ }^{85}$ once memorably called 'primitive patterns of understanding'.

That said, it should, however, be borne in mind that, in this discussion, we have deliberately eschewed versions $^{85-90}$ of the Hückel-London-Pople-McWeeny approach $^{1-4}$ in which resonance integrals between adjacent carbon-atoms are made iteratively self-consistent with respect to the corresponding calculated bondorders. We have recently re-emphasised ${ }^{13}$ that if realistic paramagnetic ring-currents are needed in systems like these - for example, for the purposes of predicting ${ }^{1} \mathrm{H}-\mathrm{NMR}$ chemical-shifts ${ }^{5-8}$ — then such an iterative calculation 'is vital'. ${ }^{13} \mathrm{~A}$ similar point has been made in Ref. 24. As in Refs. 12 and 13, however, such refinements are outside the scope of the present study, which is designed to establish how far applicability of the AWA model can be determined solely from knowledge 
of the carbon-carbon connectivity and the ring areas of a given conjugated system.

Overall, however, in our view, the beauty of the HLPM $^{1-4}$ topological $^{9-11}$ method - which, it is hoped, has been demonstrated here - is that ring-current intensities calculated by applying this formalism are entirely independent of any subjectively chosen (or, indeed, any other) parameterisations, wave functions or schemes of calculation; their numerical values are immediately predetermined (albeit only implicitly) once the carbonatom connectivities of the hydrocarbon under study, and the assumed areas of its constituent rings, have been specified.

Acknowledgements. An anonymous referee made some most helpful suggestions concerning the triplet ground-state encountered for structure No. 9 (displayed in Figure 9) and we are very grateful to Mr. C. W. Haigh for some extremely stimulating discussions and correspondence about this. We thank Mr. Colin Harris (Superintendent, Special Collections Reading Rooms) and Dr. Chris Fletcher (Keeper of Special Collections) of the Department of Special Collections and Western manuscripts, Bodleian Library, University of Oxford, for their kind help in establishing the correct reference information appropriate to the archival manuscript written by the late Professor C. A. Coulson that is cited in Ref. 53. RBM is also very grateful to the honorand of this issue, Professor Doug Klein, for his friendly and enjoyable collaboration on the subject of spanning-tree enumeration, mentioned in the Appendix.

\section{APPENDIX}

The number of spanning trees in a molecular graph such as that of coronene (with its vertices labelled as illustrated on the left-hand side of Figures 3 and 6) may be evaluated by any of the following methods:

The Matrix Tree Theorem ${ }^{64,92}$

In this approach, the matrix $\mathbf{K}$ is formed, defined as

$$
\mathbf{K}=\mathbf{D}-\mathbf{A},
$$

where (a) $\mathbf{D}$ is a diagonal matrix in which $D_{i i}=$ the degree of the vertex labelled $i$, and $D_{i j}=0$ if $i \neq j$ and (b) $\mathbf{A}$ is the vertex-adjacency matrix,${ }^{40}$ defined

$$
A_{i i}=0
$$

(ii) $\quad A_{i j}(i \neq j)=1$ if the vertices labelled $i$ and $j$ are connected by an edge

(iii) $\quad A_{i j}(i \neq j)=0$ if the vertices labelled $i$ and $j$ are not connected by an edge.

The Matrix Tree Theorem then states ${ }^{64,92}$ that the absolute size of any co-factor of $\mathbf{K}$ is equal to the number of spanning trees in the graph under study. For example, the matrix $\mathbf{K}$ for the coronene moleculargraph, labelled as in Figures 3 and 6, is

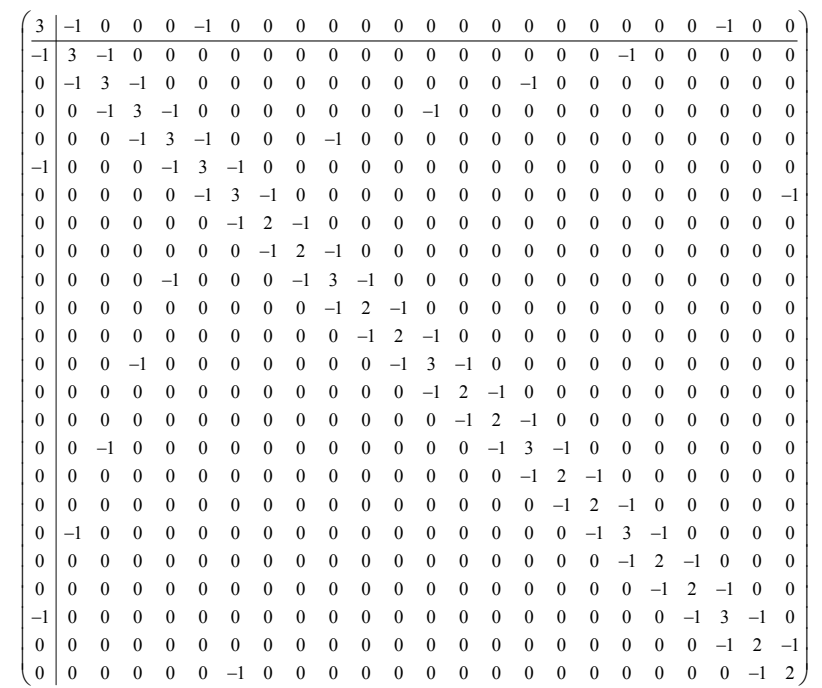

The magnitude of any co-factor of it - for example, the $(1,1)$ co-factor, obtained by deleting the first row and the first column (ruled off, as shown) - is 176400 , which is, accordingly, the number of spanning trees in the coronene molecular-graph, illustrated on the left-hand side of Figures 3 and 6.

\section{The Theorem of Gutman, Mallion \& Essam ${ }^{64}$}

Because the molecular graph of coronene is planar (in the graph-theoretical sense), it is possible to exploit the theorem of Gutman et al., ${ }^{64}$ which reduces the size of the determinant involved from one of the order of (one less than) the number of vertices in the graph (that is, in the present case, a co-factor of a $(24 \times 24)$ matrix $)$ when the Matrix-Tree Theorem is applied, to one of the order of the number of rings in the graph $-(7 \times 7)$, in the case under discussion. The process of constructing the (arbitrarily labelled) so-called 'inner-dual' ${ }^{64}$ for the coronene molecular-graph is depicted in Figure 11, and the appropriate matrix $\mathbf{G}$ that features in the theorem of Gutman et al. ${ }^{64}$ is

$$
\mathbf{G}=\left(\begin{array}{ccccccc}
6 & -1 & -1 & -1 & -1 & -1 & -1 \\
-1 & 6 & -1 & 0 & 0 & 0 & -1 \\
-1 & -1 & 6 & -1 & 0 & 0 & 0 \\
-1 & 0 & -1 & 6 & -1 & 0 & 0 \\
-1 & 0 & 0 & -1 & 6 & -1 & 0 \\
-1 & 0 & 0 & 0 & -1 & 6 & -1 \\
-1 & -1 & 0 & 0 & 0 & -1 & 6
\end{array}\right)
$$

According to the theorem, ${ }^{64}|\mathbf{G}|$ is the number of spanning trees of the original graph. Here, $|\mathbf{G}|=176400$, confirming the previous result obtained via the Matrix Tree Theorem. ${ }^{64,92}$

The Theorem of Kirby, Klein, Mallion, Pollak \& Sachs ${ }^{65}$ The theorem of Gutman et $a l^{64}$ is a special case applicable only to (graph-theoretically) planar graphs - 


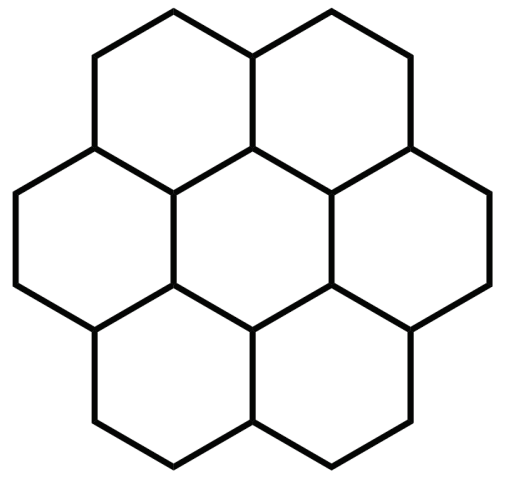

(a)

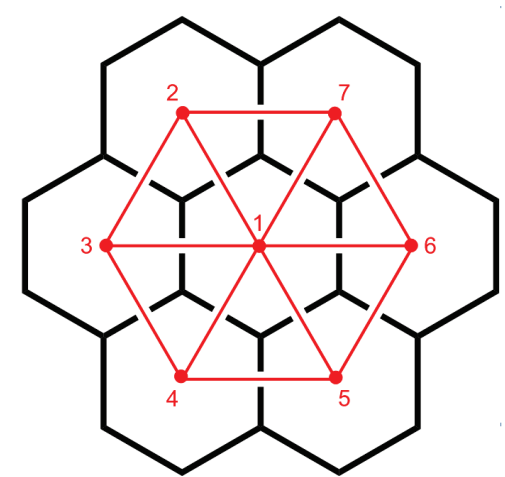

(b)

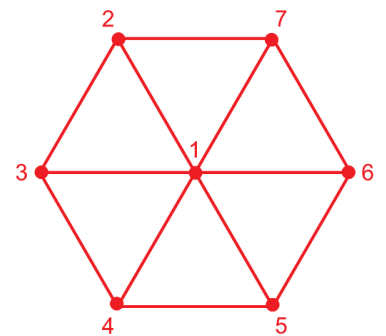

(c)

Figure 11. (a) Molecular graph of coronene; (b) process of formation of the inner dual (in red in the on-line version of the paper) of the coronene molecular-graph; (c) labelled inner-dual of the coronene molecular-graph, needed for an application of the theorem of Gutman, Mallion and Essam. ${ }^{64}$

of a more-general theorem, ${ }^{65}$ named 'the Cycle Theorem', published twenty years later by Klein (the honorand of this issue of the Croatica Chemica Acta) and collaborators (including one of the present authors $[\mathrm{RBM}])$. As has been observed, the molecular-graph of coronene (on the left-hand sides of Figures 3 and 6) is planar and it is thus possible (according to the Cycle Theorem) ${ }^{65}$ by judicious selection of an appropriate set of what are called 'patch' cycles, ${ }^{65}$ to give rise to the matrix M, featuring in the theorem of Kirby et al., ${ }^{65,93}$ which is identical with the matrix $\mathbf{G}$, just encountered in the theorem of Gutman et al. ${ }^{64}$ Since, in the Cycle Theorem, ${ }^{65,93}|\mathbf{M}|$ is (at least in the special case of the Theorem that is being considered here $)^{65,93}$ numerically equal to the number of spanning trees in the original graph, the answer of 176400 is, thereby, guaranteed, once again, to be obtained.

It should, however, be pointed out that an HLPM ring-current calculation $^{1-4}$ that capitalises on the McWeeny $^{4}$ and the Gayoso-Boucekkine ${ }^{74}$ unitarytransformations on the basis orbitals can be effected by beginning the computation starting from any spanningtree whatsoever. This means, in the case of coronene, that any one of 176400 spanning trees may be selected as the starting point. Furthermore, the remarkable fact is that, because of the myriad of relationships ${ }^{4,35,51,75}$ that exist amongst the Coulson bond-orders and the self- and mutual imaginary bond-bond polarisabilities for the 'circuit-completing' bonds, the final calculated ringcurrent intensities are independent of this overwhelming choice of starting spanning-tree that is available. No matter what spanning tree is chosen, the same ringcurrent intensities will, in the end, always result. This is why one of the present authors [RBM] has described the McWeeny method (in the pages of this journal) as 'one of the most exquisite applications of Hückel Theory'.

\section{REFERENCES}

1. C. A. Coulson, B. O'Leary, and R. B. Mallion, Hückel Theory for Organic Chemists, Academic Press, London, 1978.

2. F. London, J. Physique Radium (7ème Série) 8 (1937) 397-409.

3. J. A. Pople, Mol. Phys. 1 (1958) 175-180.

4. R. McWeeny, Mol. Phys. 1 (1958) 311-321.

5. C. W. Haigh and R. B. Mallion, Ring Current Theories in Nuclear Magnetic Resonance, in: J. W. Emsley, J. Feeney, and L. H. Sutcliffe (Eds.), Progress in Nuclear Magnetic Resonance Spectroscopy, Pergamon Press, Oxford, 1979/1980, Vol. 13, pp. 303344.

6. J. A. N. F. Gomes and R. B. Mallion, The Concept of Ring Currents, in: D. H. Rouvray (Ed.), Concepts in Chemistry, Research Studies Press Limited, Taunton, Somerset, England, United Kingdom, 1997, and John Wiley \& Sons, Inc., New York, 1997, Chapter 7, pp. 205-253.

7. P. Lazzeretti, Ring Currents, in: J. W. Emsley, J. Feeney, and L. H. Sutcliffe (Eds.), Progress in Nuclear Magnetic Resonance Spectroscopy, Elsevier, Amsterdam, 2000, Vol. 36, pp. 1-88.

8. J. A. N. F. Gomes and R. B. Mallion, Chem. Rev. 101 (2001) 1349-1383.

9. R. B. Mallion, Croat. Chem. Acta 81 (2008) 227-246.

10. A. T. Balaban, T. K. Dickens, I. Gutman, and R. B. Mallion, Croat. Chem. Acta 83 (2010) 209-215.

11. T. K. Dickens and R. B. Mallion, J. Phys. Chem. A 115 (2011) 351-356.

12. T. K. Dickens and R. B. Mallion, Phys. Chem. Chem. Phys. 15 (2013) 8245-8253.

13. T. K. Dickens and R. B. Mallion, RSC Adv. 3 (2013) 15585 15588.

14. W. E. Barth and R. G. Lawton, J. Am. Chem. Soc. 88 (1966) 380-381.

15. W. E. Barth and R. G. Lawton, J. Am. Chem. Soc. 93 (1971) 1730-1745.

16. R. Benshafrut, E. Shabtai, M. Rabinowitz, and L. T. Scott, Eur. J. Org. Chem. 6 (2000) 1091-1106.

17. E. Steiner, P. W. Fowler, L. W. Jenneskens, and A. Acocella, Chem. Commun. (2001) 659-660.

18. E. Steiner, P. W. Fowler, and L. W. Jenneskens, Angew. Chem. Int. Ed. 40 (2001) 362-366.

19. A. Acocella, R. W. A. Havenith, E. Steiner, P. W. Fowler, and L. W. Jenneskens, Chem. Phys. Letters 263 (2002) 64-72. 
20. G. Monaco, R.Viglione, R. Zanasi, and P. W. Fowler, J. Phys Chem. A 110 (2006) 7447-7452.

21. A. T. Balaban, D. E. Bean, and P. W. Fowler, Acta Chim. Slovenica 57 (2010) 507-512.

22. T. K. Dickens and R. B. Mallion, Chem. Phys. Letters $\mathbf{5 1 7}$ (2011) 98-102.

23. T. K. Dickens and R. B. Mallion, J. Phys Chem. A 115 (2011) 13877-13884.

24. G. Monaco and R. Zanasi, Phys. Chem. Chem. Phys. 15 (2013) 17654-17657.

25. D. A. Bochvar, E. G. Gal'pern, and N. P. Gambaryan, Izv. Akad. Nauk SSSR, Ser. Khim. 3 (1970) 435-437. An English translation is available from: Consultants Bureau, A Division of Plenum Publishing Corporation, 227 West 17th Street, New York, NY 10011, U.S.A., via the website http://www.springerlink.com/content/15np5207n8q10240/

26. J. Aihara, J. Am. Chem. Soc. 114 (1992) 865-868.

27. J. Aihara, J. Phys. Chem. A 112 (2008) 4382-4385.

28. Y. G. Dorfman, Diamagnetism and the Chemical Bond: C. P. Poole, (Translation Ed.), Elsevier, New York, 1965, pp. 15, 16.

29. H. C. Longuet-Higgins, Aromaticity, in: Chemical Society Special Publication No. 21; The Chemical Society: London, 1967, p. 109-111.

30. J. A. Pople and K. G. Untch, J. Am. Chem. Soc. 88 (1966) 48114815 .

31. F. Baer, H. Kuhn, and W. Regel, Z. Naturforsch. a 22 (1967) 103-112.

32. P. W. Fowler, E. Steiner, A. Acocella, L. W. Jenneskens, and R. W. A. Havenith, J. Chem. Soc. Perkin Trans 2 (2001) pp. 10581065 .

33. S. Fias, P. W. Fowler, J. L. Delgado, U. Hahn, and P. Bultinck, Chem. Eur. J. 14 (2008) 3093-3099.

34. P. W. Fowler and E. Steiner, Chem. Phys. Letters 364 (2002) 259-266.

35. R. B. Mallion, Mol. Phys. 25 (1973) 1415-1432.

36. R. B. Mallion, Proc. Royal Soc. London, Ser. A 341 (1974/1975) 429-449.

37. D. H. Rouvray, The Topological Matrix in Quantum Chemistry, in: A. T. Balaban (Ed.), Chemical Applications of Graph Theory, Academic Press, London, 1976, Chapter 7, pp. 175-221.

38. D. M. Cvetković, M. Doob, and H. Sachs, Spectra of Graphs, (a) $1^{\text {st }}$ Edition, Deutscher Verlag der Wissenschaften (East) Berlin, 1979; (b) $3^{\text {rd }}$ Edition, Johann Ambrosius Barth, Heidelberg \& Leipzig, 1995, pp. 228-238.

39. N. Trinajstić, Chemical Graph Theory, Vol. I, CRC Press, Inc., Boca Raton, Florida, U.S.A., 1983, Chapter 6, pp. 63-96.

40. D. Janežić, A. Miličević, S. Nikolić, and N. Trinajstić, Graph Theoretical Matrices in Chemistry, Mathematical Chemistry Monographs MCM 3, I. Gutman and B. Furtula, (Eds.), Faculty of Science, University of Kragejuvac, Serbia, 2007.

41. R. B. Mallion, The Topological Nature of the Aufbau Process and of Quantities Calculated via Simple Molecular-Orbital Theory, in: A. Graovac, I. Gutman, and D. Vukičević, (Eds.), Mathematical Methods and Modelling for Students of Chemistry and Biology, Hum naklada d.o.o., Zagreb, 2009, pp. 55-84.

42. L. Salem, Molecular Orbital Theory of Conjugated Systems, W. A. Benjamin, Inc., New York, 1966, Chapter 4, pp. 177-220.

43. G. Berthier, B. Pullman, and A. Pullman, Le Diamagnétisme des Composés Aromatiques, in: B. Pullman and A. Pullman (Eds), Les Théories Electroniques de la Chimie Organique, Masson, Paris, 1952, Chapter IX, pp. 527-550.

44. P. W. Fowler and W. Myrvold, J. Phys. Chem. A 115 (2011) 13191-13200.

45. B. O'Leary and R. B. Mallion, J. Math. Chem. 3 (1989) 323342.

46. C. W. Haigh and R. B. Mallion, Mol. Phys. 20 (1971) 955-970.

47. C. W. Haigh and R. B. Mallion, Organic Magnetic Resonance 4
(1972) 203-228.

48. T. K. Dickens, J. A. N. F. Gomes, and R. B. Mallion, J. Chem. Theory Computation 7 (2011) 3661-3674.

49. G. A. G. Bennett, Electricity and Modern Physics, Edward Arnold (Publishers) Ltd., London, 1968, pp. 31, 32.

50. H. M. Cundy, (Ed.) S.M.P. Advanced Mathematics Book 3 [Metric], Cambridge University Press: London \& New York, 1970, p. 911.

51. N. Mizoguchi, Bull. Chem. Soc. Jpn. 60 (1987) 2005-2010.

52. C. A. Coulson, Proc. Cambridge Philos. Soc. 36 (1940) 201203.

53. C. A. Coulson, Diamagnetic Anisotropy of Aromatics, unpublished hand-written document, dated May $16^{\text {th }}$, 1953. (Later published as a 'Special Paper' in Ref. 45). It is deposited in The Papers of Charles Alfred Coulson FRS 1910-1974 (Collection Reference CSAC 60.4.78), MS. Coulson 58 (B. 38.6), Department of Special Collections and Western Manuscripts, Bodleian Library, University of Oxford.

54. J. Aihara, J. Am. Chem. Soc. 101 (1979) 5913-5917.

55. J. Aihara and T. Horikawa, Bull. Chem. Soc. Jpn. 56 (1983) 1853-1854.

56. J. Aihara, J. Am. Chem. Soc. 107 (1985) 298-302.

57. J. Aihara, R. Sekine, and S. Oe, Croat. Chem. Acta 77 (2004) 353-359.

58. R. B. Mallion and D. H. Rouvray, Mol. Phys. 36 (1978) 125 128 .

59. R. B. Mallion and D. H. Rouvray, Studia Scientiarum et Mathematicarum Hungarica 13 (1978) 229-243.

60. A. Ceulemans, E. Lijnen, P. W. Fowler, R. B. Mallion, and T. Pisanski, Proc. Royal Soc. London Ser. A 468 (2012) 971-989.

61. C. A. Coulson, Proc. Royal Soc. London Ser. A 169 (1938/1939) $413-428$.

62. C. A. Coulson and H. C. Longuet-Higgins, Proc. Royal Soc. London Ser. A 191 (1947) 39-60.

63. R. J. Wilson, Introduction to Graph Theory, $1^{\text {st }}$ Edition, Oliver \& Boyd, Edinburgh, 1972, (a) pp. 36-38 \& 46, 47; (b) p. 53.

64. I. Gutman, R. B. Mallion, and J. W. Essam, Mol. Phys. 50 (1983) 859-877.

65. E. C. Kirby, D. J. Klein, R. B. Mallion, P. Pollak, and H. Sachs, Croat. Chem. Acta 77 (2004), 263-278.

66. F. Harary and R. B. Mallion, Nanta Mathematica 7 (1974) 96101.

67. 'S.' Kirchhoff, Annal. Phys. Chem. ('Poggendorf's Annalen') 64 (1845) 497-514.

68. G. Kirchhoff, Annal. Phys. Chem. ('Poggendorf's Annalen') 72 (1847) 497-508

69. A. Veillard, J. Chim. Phys. Phys.-Chim. Biol. 59 (1962) 10561066.

70. R. B. Mallion, J. Chem. Soc. Perkin Trans. II (1973), pp. 235 237.

71. R. B. Mallion, Biochimie 56 (1974) 187-188.

72. I. J. Maddox and R. McWeeny, J. Chem. Phys. 36 (1962) $2353-$ 2354.

73. N. Jonathan, S. Gordon, and B. P. Dailey, J. Chem. Phys. 36 (1962) 2443-2448.

74. J. Gayoso and A. Boucekkine, C. R. Hebd. Séances Acad. Sci. Paris Sér. C 272 (1971) 184-187.

75. C. W. Haigh and R. B. Mallion, Croat. Chem. Acta 62 (1989) 126.

76. C. W. Haigh, R. B. Mallion, and E. A. G. Armour, Mol. Phys. 18 (1970) 751-766.

77. R. B. Mallion, J. Mol. Spectrosc. 35 (1970) 491-493.

78. I. Agranat, B. A. Hess, and L. J. Schaad, Pure Applied Chem. 52 (1980) 1399-1407.

79. L. Pauling, L. O. Brockway, and J. W. Beach, J. Am. Chem. Soc. 57 (1935) 2705-2709.

80. S. J. Cyvin and I. Gutman, Lecture Notes in Chemistry 46, 
Kekulé Structures in Benzenoid Hydrocarbons, Springer-Verlag, Berlin (West) \& Heidelberg, 1988, p.5.

81. R. B. Mallion and D. H. Rouvray, J. Math. Chem. 5 (1990) 1-21; see also 'Postscript' 8 (1991) 399-400.

82. M. Randić, M. Nović, M. Vračko, D. Vukičević, and D. Plavšić, Int. J. Quantum Chem. 112 (2012) 972-985.

83. M. Randić, Chem. Phys. Letters 500 (2010) 123-127.

84. J. A. N. F. Gomes and R. B. Mallion, Revista Portuguesa de Química 21 (1979) 82-89.

85. C. A. Coulson, Rev. Mod. Phys. 32 (1960) 170-177.

86. C. A. Coulson, J. A. N. F. Gomes, and R. B. Mallion, Mol. Phys. 30 (1975) 713-732.

87. C. A. Coulson and R. B. Mallion, J. Am. Chem. Soc. 98 (1976) 592-598.
88. R. B. Mallion, Pure Appl. Chem. 52 (1980) 1541-1548.

89. J. A. N. F. Gomes and R. B. Mallion, J. Org. Chem. 46 (1981) 719-727.

90. C. F. Wilcox and E. N. Farley, J. Am. Chem. Soc. 106 (1984) 7195-7200.

91. R. B. Mallion, Nature 325 (1987) 760-761.

92. R. B. Mallion, Chem. Phys. Letters 36 (1975) 170-174.

93. E. C. Kirby, R. B. Mallion, and P. Pollak, Counting Spanning Trees in Toroidal Fullerenes, in: F. Cataldo and P. Miliani, (Series Eds.) Carbon Materials: Chemistry and Physics: F. Cataldo, A. Graovac and O. Ori (Eds.) Volume 4: The Mathematics and Topology of Fullerenes, Springer, Dordrecht, Heidelberg, London \& New York, 2011, Chapter 10, pp. 187-204. 